Article

\title{
The Risk Measurement under the Variance-Gamma Process with Drift Switching
}

\author{
Roman V. Ivanov
}

check for updates

Citation: Ivanov, Roman V. 2022. The Risk Measurement under the Variance-Gamma Process with Drift Switching. Journal of Risk and Financial Management 15: 22. https://doi.org/10.3390/ jrfm15010022

Academic Editor: Thanasis Stengos

Received: 9 December 2021

Accepted: 5 January 2022

Published: 7 January 2022

Publisher's Note: MDPI stays neutral with regard to jurisdictional claims in published maps and institutional affiliations.

Copyright: (C) 2022 by the author. Licensee MDPI, Basel, Switzerland. This article is an open access article distributed under the terms and conditions of the Creative Commons Attribution (CC BY) license (https:// creativecommons.org/licenses/by/ $4.0 /)$.
Laboratory of Control under Incomplete Information, V.A. Trapeznikov Institute of Control Sciences of RAS, Profsoyuznaya 65, 117997 Moscow, Russia; roivanov@yahoo.com

\begin{abstract}
The paper discusses an extension of the variance-gamma process with stochastic linear drift coefficient. It is assumed that the linear drift coefficient may switch to a different value at the exponentially distributed time. The size of the drift jump is supposed to have a multinomial distribution. We have obtained the distribution function, the probability density function and the lower partial expectation for the considered process in closed forms. The results are applied to the calculation of the value at risk and the expected shortfall of the investment portfolio in the related multivariate stochastic model.
\end{abstract}

Keywords: variance-gamma process; drift switching; exponential distribution; hypergeometric function; lower partial expectation; value at risk; expected shortfall

\section{Introduction}

The variance-gamma distribution determines a well-known model of the financial data evolution. The symmetric variant of the variance-gamma distribution was studied in Madan and Seneta (1990); Madan and Milne (1991). Madan et al. (1998) defined the variance-gamma process as the time-changed Brownian motion with drift. A number of modern research papers confirms statistically the idea to use the variance-gamma distribution for the financial index modeling by the statistical analysis of the stock market data. Daal and Madan (2005); Finlay and Seneta (2006) approve the variance-gamma model for the exchange rate simulation. Linders and Stassen (2016); Moosbrucker (2006); Rathgeber et al. (2016) model with the variance-gamma distribution the Dow Jones index returns. Mozumder et al. (2015) consider the S\&P500 index options in the variancegamma model. Luciano and Schoutens (2016) model the S\&P500, the Nikkei225 and the Eurostoxx50 financial indices by the variance-gamma process. Luciano et al. (2016); Wallmeier and Diethelm (2012) confirm the use of the variance-gamma distribution for the modeling of the US and the Swiss stock markets, respectively. Groups of various financial indices are modeled by the multivariate variance-gamma distribution in Nitithumbundit and Chan (2020). Flora and Vargioulu (2020) find that the variance-gamma process is the best fit for the carbon price dynamics. Göncü et al. (2016) show that the variance-gamma model fits well with the financial data of developed markets. Hoyyi et al. (2021) argue for the use of the variance-gamma distribution for the return modeling on the Southeast Asian stock markets.

There is a number of theoretical papers on the variance-gamma process which mostly investigate the computation problems connected to it. Madan et al. (1998); Ano and Ivanov (2016); Ivanov (2021) establish closed-form results for the option prices and the values of the monetary risk measures. Avramidis et al. (2003); Fu (2007) suggest special Monte-Carlo type procedures. The Fourier transform methodology for the variance-gamma model was developed in Carr and Madan (1999); Almendral and Oosterlee (2007). A calculation method based on the Mellin transform is proposed in Aguilar (2020). The triple Mellin-Barnes integral representation for the European call price is given in Febrer 
and Guerra (2021). It is assumed in the all studies above that the drift coefficient of the underlying variance-gamma distribution is constant. However, the drift coefficient may switch because of Federal Reserve announcements. This problem is considered in the papers by Cook and Hahn (1989), by Bernanke and Blinder (1992) and by Nakamura and Steinsson (2018). The calculation of the value at risk and the expected shortfall in better economically based models helps to improve the backtesting results, see Christoffersen and Pelletier (2004). Furthermore, the computation of these two basic monetary risk measures can allow us to pass to the advanced risk measurement, see the works by Barone Adesi (2016) and by Keçeci et al. (2016).

If we suggest that the drift coefficient might change, then the usual choice for its time modeling is the exponential distribution. This type of the drift switching was discussed for the Brownian motion particularly in the works by Shiryaev (1963); Beibel and Lerche (1997); Novikov and Shiryaev (2009) for the quickest detection problem. The calculation of option prices in discrete time models under the regime switching is discussed in Rèmillard et al. (2017). The geometric Brownian motion with the drift and volatility switching is considered for various computational problems in the papers by Fuh et al. (2012); Yao et al. (2006); Zhang and Zhou (2009). Some limit results for jump-diffusions with regime switching are presented in Yin and Zhu (2010).

\section{Materials and Methods}

We discuss in this paper an extension of the variance-gamma process assuming that the linear drift coefficient may spontaneously change. From the economical point of view such modeling can take into account a possible fluctuation of the target federal funds rate. Changes in the federal funds rate can affect other short-term interest rates, longer-term interest rates, foreign exchange rates, stock prices and the prices of goods and services as well. Probably the most known works on this topic are the papers by Cook and Hahn (1989) and by Bernanke and Blinder (1992). Among the modern works in this direction let us mention the paper by Nakamura and Steinsson (2018) where the impact of Federal Reserve announcements on the whole economy is studied.

Similarly to Shiryaev (1963), the time of the linear drift change is supposed to be exponentially distributed. The distribution of the linear drift coefficient at the time of the change is suggested to be multinomial. Section 4 summarizes the properties of the new process, introduces its distribution and probability density functions. Furthermore, we introduce in Section 4 a formula for the lower partial expectation. Section 5 applies the results to the computation of the value at risk and the expected shortfall monetary risk measures. The paper proceeds the direction of research of the works by Madan et al. (1998); Ano and Ivanov (2016); Ivanov (2018); Ivanov and Temnov (2016) where analytical results were obtained in the variance-gamma model and its generalizations.

\section{Setup and Notations}

\subsection{Setup}

Let $\gamma_{t}=\gamma_{t}(a), t \geq 0, a>0$, be a gamma process with unit mean from zero. It is the purely discontinuous Lévy process which has the probability density function

$$
f\left(\gamma_{t}, x\right)=\frac{a^{a t} x^{a t-1} e^{-a x}}{\Gamma(a t)}, \quad t>0, x>0,
$$

where $\Gamma(u), u>0$, is the gamma function. The variance-gamma process $X_{t}^{v g}$ is defined as

$$
X_{t}^{v g}=\mu t+\theta \gamma_{t}+\sigma B_{\gamma_{t}}
$$


where $\mu, \theta \in \mathbb{R}, \sigma>0$ are constants, $B_{s}, s \geq 0$, is the Brownian motion with $B_{0}=0$ and $\gamma_{t}$ is the independent with the Brownian motion gamma process with the density (1). The variance-gamma process has the mean

$$
\mathrm{E} X_{t}^{v g}=(\mu+\theta) t
$$

the variance

$$
\operatorname{Var} X_{t}^{v g}=\left(\frac{\theta^{2}}{a}+\sigma^{2}\right) t
$$

and the characteristic function

$$
\varphi_{X_{t}^{v g}}(u)=e^{i u \mu t}\left(1-\frac{i u \theta}{a}+\frac{(\sigma u)^{2}}{2 a}\right)^{-a t},
$$

see for example Madan et al. (1998).

Throughout this paper, we discuss a generalization of the process (2). It is assumed that the linear drift rate can decrease by a jump at a moment $\tau \geq 0$ from $\mu$ to some value between $\mu$ and $\mu_{1}, \mu_{1} \leq \mu$. That is, we consider the process

$$
X_{t}=\int_{0}^{t} \mu(s) d s+\theta \gamma_{t}+\sigma B_{\gamma_{t}}
$$

with $\theta \in \mathbb{R}, \sigma>0$, the Brownian motion $B_{s}, s \geq 0, B_{0}=0$, the independent with the Brownian motion gamma process $\gamma_{t}$ with the density (1), $\gamma_{0}=0$, and

$$
\mu(s)=\mu I_{\{\tau>s\}}+\widehat{\mu} I_{\{\tau \leq s\}}, \quad s \geq 0,
$$

where the random variable $\widehat{\mu}$ has the multinomial distribution with

$$
\widehat{\mu} \in\left\{\mu_{1}, \mu_{2}, \ldots, \mu_{m}, \mu\right\}
$$

and

$$
\mu_{1} \leq \mu_{2} \leq \cdots \leq \mu_{m} \leq \mu
$$

It is assumed that

$$
\mathrm{P}\left(\widehat{\mu}=\mu_{j}\right)=\mathrm{p}_{j} \geq 0, \quad \mathrm{P}(\widehat{\mu}=\mu)=\mathrm{p} \geq 0, \quad \mathrm{p}+\sum_{j=1}^{m} \mathrm{p}_{j}=1 .
$$

The random variable $\tau$ is suggested to be exponentially distributed with a parameter $\lambda>0$. It can be noticed easily that then

$$
\int_{0}^{t} \mu(s) d s=(\mu \tau+\widehat{\mu}(t-\tau)) I_{\{\tau \leq t\}}+\mu t I_{\{\tau>t\}} .
$$

It is supposed also that $\tau, \widehat{\mu}$ are independent between each other and with $B_{s}$ and $\gamma_{t}$.

\subsection{Special Notations}

We set

$$
\operatorname{sgn}(u)= \begin{cases}1 & \text { if } u>0 \\ 0 & \text { if } u=0 \\ -1 & \text { if } u<0\end{cases}
$$

and use notations 


$$
\Psi(u), u \in \mathbb{R}, \quad \mathrm{B}\left(u_{1}, u_{2}\right), u_{1}>0, u_{2}>0, \quad \mathrm{~K}_{u_{1}}\left(u_{2}\right), u_{1} \in \mathbb{R}, u_{2}>0
$$

for the normal distribution function, the beta function and the MacDonald function (the modified Bessel function of the second kind), respectively. The hypergeometric Gauss function is denoted as

$$
\mathrm{F}\left(u_{1}, u_{2}, u_{3} ; u_{4}\right), u_{1}, u_{2}, u_{3} \in \mathbb{R}, u_{4}<1 \text {. }
$$

Furthermore, the degenerate Appell functions (or the Humbert series) which is the double sum

$$
\Phi\left(u_{1}, u_{2}, u_{3} ; u_{4}, u_{5}\right)=\sum_{m=0}^{\infty} \sum_{n=0}^{\infty} \frac{\left(u_{1}\right)_{m+n}\left(u_{2}\right)_{m}}{m ! n !\left(u_{3}\right)_{m+n}} u_{4}^{m} u_{5}^{n}
$$

with $u_{1}, u_{2}, u_{3}, u_{5} \in \mathbb{R}$ and $\left|u_{4}\right|<1$, where $(u)_{l}, l \in \mathbb{N} \cup\{0\}$, is the Pochhammer's symbol, is exploited. For more information on the special mathematical functions above, see the monographs by Bateman and Erdélyi (1953); Srivastava and Karlsson (1985) and the handbook by Gradshteyn and Ryzhik (2007).

Basing on the definitions of the special functions above, let us introduce supplementary functions

$$
\mathfrak{L}\left(u_{1}, u_{2}\right), \quad \mathfrak{I}_{=}\left(\alpha, u_{1}, u_{2}\right), \quad \mathfrak{I}_{>}\left(\alpha, u_{1}, u_{2}, u_{3}\right), \quad \mathfrak{I}_{<}\left(\alpha, u_{1}, u_{2}, u_{3}\right) .
$$

We set for $t>0$

$$
\begin{aligned}
& \mathfrak{L}\left(u_{1}, u_{2}\right)=\frac{a^{a t} e^{\frac{\theta u_{2}}{\sigma^{2}} \sqrt{2}}}{\sigma \Gamma(a t) \sqrt{\pi}}\left(\frac{\left|u_{2}\right|}{\sqrt{2 a \sigma^{2}+\theta^{2}}}\right)^{a t-\frac{1}{2}} \times \\
& \times \mathrm{K}_{a t-\frac{1}{2}}\left(\frac{\left|u_{2}\right| \sqrt{2 a \sigma^{2}+\theta^{2}}}{\sigma^{2}}\right)\left[I_{\left\{u_{1}=0, u_{2} \neq 0\right\}}+e^{u_{1} u_{2}} I_{\left\{u_{1} \neq 0, u_{2} \neq 0\right\}}\right]+ \\
&+\frac{a^{a t} \Gamma\left(a t-\frac{1}{2}\right)}{\sigma\left(a+\frac{\theta^{2}}{2 \sigma^{2}}\right)^{a t-\frac{1}{2}} \Gamma(a t) \sqrt{2 \pi}} I_{\left\{u_{2}=0, t>\frac{1}{2 a}\right\}}+\infty I_{\left\{u_{2}=0, t \leq \frac{1}{2 a}\right\}},
\end{aligned}
$$

where $u_{1}, u_{2} \in \mathbb{R}$. Let

$$
\begin{aligned}
& \mathfrak{I}_{=}\left(\alpha, u_{1}, u_{2}\right)=\frac{2^{\alpha} \Gamma\left(\alpha+\frac{3}{2}\right)}{(\alpha+1)\left|u_{1}\right|^{2(\alpha+1)} \sqrt{\pi}} I_{\left\{u_{2}=0\right\}}+\frac{e^{u_{1} u_{2}}\left|u_{2}\right|^{\alpha+\frac{3}{2}}}{(\alpha+1) \sqrt{2 \pi}\left|u_{1}\right|^{\alpha+\frac{1}{2}}} \times \\
& \times\left[\operatorname{sgn}\left(u_{2}\right) \mathrm{K}_{\alpha+\frac{1}{2}}\left(\left|u_{1} u_{2}\right|\right)+\mathrm{K}_{\alpha+\frac{3}{2}}\left(\left|u_{1} u_{2}\right|\right)\right] I_{\left\{u_{2} \neq 0\right\}}
\end{aligned}
$$

for $\alpha>-1, u_{2} \in \mathbb{R}$ and $u_{1} \neq 0$. The function

$$
\begin{aligned}
& \mathfrak{I}_{>}\left(\alpha, u_{1}, u_{2}, u_{3}\right)= \\
= & \frac{\Gamma\left(\alpha+\frac{3}{2}\right)}{\sqrt{2 \pi}\left(a-u_{1}\right)^{\alpha+1}}\left[\frac{\mathrm{B}\left(\frac{1}{2}, \alpha+1\right)}{\sqrt{2}}+\frac{u_{2}}{\sqrt{a-u_{1}}} \mathrm{~F}\left(\alpha+\frac{3}{2}, \frac{1}{2}, \frac{3}{2} ; \frac{u_{2}^{2}}{2\left(u_{1}-a\right)}\right)\right] I_{\left\{u_{3}=0\right\}} \\
+ & \frac{\left|s_{>}\right|^{\alpha+\frac{1}{2}} e^{s_{>}}(1+q>)^{\alpha+1}}{\sqrt{2 \pi}\left(a-u_{1}\right)^{\alpha+1}}\left[\mathrm{~B}(\alpha+1,1)\left(\left|s_{>}\right| \mathrm{K}_{\alpha+\frac{3}{2}}\left(\left|s_{>}\right|\right)+s_{>} \mathrm{K}_{\alpha+\frac{1}{2}}\left(\left|s_{>}\right|\right)\right)\right. \\
\times & \Phi\left(\alpha+1,-\alpha, \alpha+2 ; \frac{1+q>}{2},-s_{>}\left(1+q_{>}\right)\right)-(1+q>) s_{>} \mathrm{B}(\alpha+2,1) \times \\
\times & \left.\mathrm{K}_{\alpha+\frac{1}{2}}\left(\left|s_{>}\right|\right) \Phi\left(\alpha+2,-\alpha, \alpha+3 ; \frac{1+q>}{2},-s_{>}\left(1+q_{>}\right)\right)\right] I_{\left\{u_{3} \neq 0\right\}},
\end{aligned}
$$


is defined for $a>u_{1}, u_{2}, u_{3} \in \mathbb{R}$ and $\alpha>-1$ with

$$
q>=\frac{u_{2}}{\sqrt{u_{2}^{2}+2\left(a-u_{1}\right)}} \quad \text { and } \quad s_{>}=u_{3} \sqrt{u_{2}^{2}+2\left(a-u_{1}\right)} .
$$

Further, set

$$
\begin{aligned}
& \mathfrak{I}_{<}\left(\alpha, u_{1}, u_{2}, u_{3}\right)= \\
= & \frac{2^{\alpha} \Gamma\left(\alpha+\frac{3}{2}\right)}{(\alpha+1)\left(u_{2}^{2}-2\left(u_{1}-a\right)\right)^{\alpha+1} \sqrt{\pi}} \mathrm{F}\left(\frac{1}{2}, \alpha+1, \alpha+2 ; \frac{2\left(u_{1}-a\right)}{2\left(u_{1}-a\right)-u_{2}^{2}}\right) I_{\left\{u_{3}=0\right\}}+ \\
+ & \frac{(q<-1)^{\alpha+1}\left|s_{<}\right|^{\alpha+\frac{1}{2}} e^{s<}}{\left(u_{1}-a\right)^{\alpha+1} \sqrt{2 \pi}}\left[\mathrm{B}(\alpha+1,1) \Phi\left(\alpha+1,-\alpha, \alpha+2 ; \frac{1-q_{<}}{2}, s_{<}\left(1-q_{<}\right)\right)\right. \\
\times & \left(\left|s_{<}\right| \mathrm{K}_{\alpha+\frac{3}{2}}\left(\left|s_{<}\right|\right)+s_{<} \mathrm{K}_{\alpha+\frac{1}{2}}\left(\left|s_{<}\right|\right)\right)+(q<-1) s_{<} \mathrm{K}_{\alpha+\frac{1}{2}}\left(\left|s_{<}\right|\right) \mathrm{B}(\alpha+2,1) \\
\times & \left.\Phi\left(\alpha+2,-\alpha, \alpha+3 ; \frac{1-q_{<}}{2}, s_{<}\left(1-q_{<}\right)\right)\right] I_{\left\{u_{3} \neq 0\right\}}
\end{aligned}
$$

for $a<u_{1}, u_{2} \geq \sqrt{2 u_{1}}, u_{3} \in \mathbb{R}$ and $\alpha>-1$ with

$$
q_{<}=-\frac{u_{2}}{\sqrt{u_{2}^{2}-2\left(u_{1}-a\right)}} \quad \text { and } \quad s_{<}=u_{3} \sqrt{u_{2}^{2}-2\left(u_{1}-a\right)} .
$$

Finally, let

$$
z_{j}=\frac{\lambda \sigma}{\mu_{j}-\mu}-\frac{\theta}{\sigma}
$$

and

$$
w_{j}=\frac{\lambda}{\mu-\mu_{j}}\left(\theta+\frac{\lambda \sigma^{2}}{2\left(\mu-\mu_{j}\right)}\right)
$$

for $j=1,2, \ldots, m$.

\section{Theoretical Results}

In this section, we discuss the variance-gamma process with drift switching which is defined in (6). Using (7), one can observe that

$$
X_{t}=(\mu \tau+\widehat{\mu}(t-\tau)) I_{\{\tau \leq t\}}+\mu t I_{\{\tau>t\}}+\theta \gamma_{t}+\sigma B_{\gamma_{t}} .
$$

At first, we compute the mean, the variance and the characteristic function of the process $X_{t}$.

\subsection{Properties}

We have that the mean of $X_{t}$

$$
\begin{aligned}
\mathrm{E} X_{t} & =\theta t+\mathrm{E}\left((\mu \tau+\widehat{\mu}(t-\tau)) I_{\{\tau \leq t\}}+\mu t I_{\{\tau>t\}}\right)= \\
& =\theta t+\mu t e^{-\lambda t}+\mu \mathrm{E}\left(\tau I_{\{\tau \leq t\}}\right)+\left(\mathrm{p} \mu+\sum_{j=1}^{m} \mathrm{p}_{j} \mu_{j}\right) \times \\
& \times\left(t\left(1-e^{-\lambda t}\right)-\mathrm{E}\left(\tau I_{\{\tau \leq t\}}\right)\right) .
\end{aligned}
$$


Since

$$
\mathrm{E}\left(\tau I_{\{\tau \leq t\}}\right)=\lambda \int_{0}^{t} x e^{-\lambda x} d x=\frac{1}{\lambda}\left(1-e^{-\lambda t}\right)-t e^{-\lambda t}
$$

we get that

$$
\mathrm{EX}_{t}=\theta t+\frac{\mu}{\lambda}\left(1-e^{-\lambda t}\right)+\left(\mathrm{p} \mu+\sum_{j=1}^{m} \mathrm{p}_{j} \mu_{j}\right)\left(t-\frac{1}{\lambda}\left(1-e^{-\lambda t}\right)\right)
$$

with

$$
\mathrm{E} \widehat{\mu}=\mathrm{p} \mu+\sum_{j=1}^{m} \mathrm{p}_{j} \mu_{j}
$$

If $\mu=\mu_{1}=\cdots=\mu_{m}$, we have (3) immediately from (15).

Further, the expectation

$$
\begin{aligned}
& \mathrm{E} X_{t}^{2}=\mu^{2} \mathrm{E}\left(\tau^{2} I_{\{\tau \leq t\}}\right)+\mu \mathrm{E}\left(\widehat{\mu} \tau(t-\tau) I_{\{\tau \leq t\}}\right)+\mu \theta \mathrm{E}\left(\gamma_{t} \tau I_{\{\tau \leq t\}}\right)+ \\
& +\mu \sigma \mathrm{E}\left(B_{\gamma_{t}} \tau I_{\{\tau \leq t\}}\right)+\mu \mathrm{E}\left(\widehat{\mu} \tau(t-\tau) I_{\{\tau \leq t\}}\right)+\mathrm{E}\left(\widehat{\mu}^{2}(t-\tau)^{2} I_{\{\tau \leq t\}}\right)+ \\
& +\theta \mathrm{E}\left(\widehat{\mu} \gamma_{t}(t-\tau) I_{\{\tau \leq t\}}\right)+\sigma \mathrm{E}\left(\widehat{\mu} B_{\gamma_{t}}(t-\tau) I_{\{\tau \leq t\}}\right)+\mu^{2} t^{2} e^{-\lambda t}+ \\
& +\mu \theta t^{2} e^{-\lambda t}+\theta t\left(\mathrm{E} X_{t}-\theta t\right)+\theta^{2} \mathrm{E} \gamma_{t}^{2}+\sigma^{2} \mathrm{E} B_{\gamma_{t}}^{2}=\mu^{2} \mathrm{E}\left(\tau^{2} I_{\{\tau \leq t\}}\right)+ \\
& +2 \mu t \mathrm{E} \widehat{\mu} \mathrm{E}\left(\tau I_{\{\tau \leq t\}}\right)-2 \mu \mathrm{E} \widehat{\mu} \mathrm{E}\left(\tau^{2} I_{\{\tau \leq t\}}\right)+\mu \theta t \mathrm{E}\left(\tau I_{\{\tau \leq t\}}\right)+ \\
& +t^{2} \mathrm{E} \widehat{\mu}^{2}\left(1-e^{-\lambda t}\right)-2 t \mathrm{E} \widehat{\mu}^{2} \mathrm{E}\left(\tau I_{\{\tau \leq t\}}\right)+\mathrm{E} \widehat{\mu}^{2} \mathrm{E}\left(\tau^{2} I_{\{\tau \leq t\}}\right)+ \\
& +\theta t^{2}\left(1-e^{-\lambda t}\right) \mathrm{E} \widehat{\mu}-\theta t \mathrm{E} \widehat{\mu} \mathrm{E}\left(\tau I_{\{\tau \leq t\}}\right)+\mu^{2} t^{2} e^{-\lambda t}+\mu \theta t^{2} e^{-\lambda t}+ \\
& +\theta t\left(\mathrm{E} X_{t}-\theta t\right)+\theta^{2} t\left(t+\frac{1}{a}\right)+\sigma^{2} t=\mathrm{E}\left(\tau^{2} I_{\{\tau \leq t\}}\right)\left(\mu^{2}-2 \mu \mathrm{E} \widehat{\mu}+\mathrm{E} \widehat{\mu}^{2}\right)+ \\
& +\mathrm{E}\left(\tau I_{\{\tau \leq t\}}\right)\left(2 \mu t \mathrm{E} \widehat{\mu}+\mu \theta t-2 t \mathrm{E} \widehat{\mu}^{2}-\theta t \mathrm{E} \widehat{\mu}\right)+t^{2}\left(1-e^{-\lambda t}\right) \times \\
& \times\left(\mathrm{E} \widehat{\mu}^{2}+\theta \mathrm{E} \widehat{\mu}\right)+t^{2} \mu e^{-\lambda t}(\mu+\theta)+t\left(\theta \mathrm{E} X_{t}+\frac{\theta^{2}}{a}+\sigma^{2}\right) .
\end{aligned}
$$

Because of

$$
\begin{aligned}
\mathrm{E}\left(\tau^{2} I_{\{\tau \leq t\}}\right) & =\lambda \int_{0}^{t} x^{2} e^{-\lambda x} d x=-t^{2} e^{-\lambda t}+2 \int_{0}^{t} x e^{-\lambda x} d x= \\
& =\frac{2}{\lambda} \mathrm{E}\left(\tau I_{\{\tau \leq t\}}\right)-t^{2} e^{-\lambda t}
\end{aligned}
$$

we get that the variance of $X_{t}$

$$
\begin{aligned}
& \operatorname{Var} X_{t}=\left(\frac{2}{\lambda} \mathrm{E}\left(\tau I_{\{\tau \leq t\}}\right)-t^{2} e^{-\lambda t}\right)\left(\mu^{2}-2 \mu \mathrm{E} \widehat{\mu}+\mathrm{E} \widehat{\mu}^{2}\right)+ \\
& +\mathrm{E}\left(\tau I_{\{\tau \leq t\}}\right)\left(2 \mu t \mathrm{E} \widehat{\mu}+\mu \theta t-2 t \mathrm{E} \widehat{\mu}^{2}-\theta t \mathrm{E} \widehat{\mu}\right)+t^{2}\left(1-e^{-\lambda t}\right) \times \\
& \times\left(\mathrm{E} \widehat{\mu}^{2}+\theta \mathrm{E} \widehat{\mu}\right)+t^{2} \mu e^{-\lambda t}(\mu+\theta)+t\left(\theta \mathrm{E} X_{t}+\frac{\theta^{2}}{a}+\sigma^{2}\right)-\left(\mathrm{E} X_{t}\right)^{2},
\end{aligned}
$$


where $\mathrm{E}\left(\tau I_{\{\tau \leq t\}}\right), \mathrm{E} X_{t}, \mathrm{E} \widehat{\mu}$ are determined by (14), (15), (16), respectively, and

$$
\mathrm{E} \widehat{\mu}^{2}=\mathrm{p} \mu^{2}+\sum_{j=1}^{m} \mathrm{p}_{j} \mu_{j}^{2}
$$

Setting $\mu=\mu_{1}=\cdots=\mu_{m}$, we establish from (17) the variance (4).

Next, the expectation

$$
\begin{aligned}
\mathrm{E}^{i u \int_{0}^{t} \mu(s) d s} & =\mathrm{E}\left(e^{i u(\mu \tau+\widehat{\mu}(t-\tau))} I_{\{\tau \leq t\}}\right)+e^{(i u \mu-\lambda) t}=\mathrm{p} e^{i u \mu t}\left(1-e^{-\lambda t}\right)+ \\
& +e^{(i u \mu-\lambda) t}+\sum_{j=1}^{m} \mathrm{p}_{j} \mathrm{E}\left(e^{i u\left(\mu \tau+\mu_{j}(t-\tau)\right)} I_{\{\tau \leq t\}}\right)
\end{aligned}
$$

and since

$$
\begin{aligned}
& \mathrm{E}\left(e^{i u \tau\left(\mu-\mu_{j}\right)} I_{\{\tau \leq t\}}\right)=\lambda \int_{0}^{t} e^{-\lambda x+i u x\left(\mu-\mu_{j}\right)} d x= \\
& =\lambda \int_{0}^{t} e^{-\lambda x}\left(\cos u\left(\mu-\mu_{j}\right) x+i \sin u\left(\mu-\mu_{j}\right) x\right) d x= \\
& =u\left(\mu-\mu_{j}\right) \int_{0}^{t} e^{-\lambda x}\left(i \cos u\left(\mu-\mu_{j}\right) x-\sin u\left(\mu-\mu_{j}\right) x\right) d x- \\
& e^{-\lambda t+i u t\left(\mu-\mu_{j}\right)}+1=1+\frac{i u\left(\mu-\mu_{j}\right)}{\lambda} \mathrm{E}\left(e^{i u \tau\left(\mu-\mu_{j}\right)} I_{\{\tau \leq t\}}\right)-e^{-\lambda t+i u t\left(\mu-\mu_{j}\right),}
\end{aligned}
$$

we get that

$$
\begin{aligned}
& \mathrm{E} e^{i u \int_{0}^{t} \mu(s) d s}= \\
= & \mathrm{p} e^{i u \mu t}\left(1-e^{-\lambda t}\right)+e^{(i u \mu-\lambda) t}+\sum_{j=1}^{m} \mathrm{p}_{j} e^{i u \mu_{j} t}\left(\frac{\lambda\left(1-e^{t\left(i u\left(\mu-\mu_{j}\right)-\lambda\right)}\right)}{\lambda-i u\left(\mu-\mu_{j}\right)}\right) .
\end{aligned}
$$

Hence the characteristic function of $X_{t}$

$$
\begin{aligned}
& \varphi_{X_{t}}(u)=\left(\mathrm{E}^{i u\left(X_{t}^{v g}-\mu t\right)}\right)\left(\mathrm{E}^{i u \int_{0}^{t} \mu(s) d s}\right)=\left(1-\frac{i u \theta}{a}+\frac{(\sigma u)^{2}}{2 a}\right)^{-a t} \times \\
& \times\left(\mathrm{p} e^{i u \mu t}\left(1-e^{-\lambda t}\right)+e^{(i u \mu-\lambda) t}+\sum_{j=1}^{m} \mathrm{p}_{j} e^{i u \mu_{j} t}\left(\frac{\lambda\left(1-e^{t\left(i u\left(\mu-\mu_{j}\right)-\lambda\right)}\right)}{\lambda-i u\left(\mu-\mu_{j}\right)}\right)\right) .
\end{aligned}
$$

When $\mu=\mu_{1}=\cdots=\mu_{m}$, we immediately have (5) from (18)

\subsection{Main Results}

Now we pass to the formulation of the main results of the work. Let us define the function $\mathfrak{V}$ for $v_{2} \neq 0$ as

$$
\begin{aligned}
& \mathfrak{V}=\mathfrak{V}\left(v_{1}, v_{2}, v_{3}, v_{4}, d_{j}, g_{j}, h_{j}, c_{j}, u ; j=1, \ldots, v_{4}\right)=\frac{a^{a t}}{\Gamma(a t)} \times \\
& \times\left(\mathfrak{I}\left(a t-1,0,-\frac{v_{1}}{v_{2}}, \frac{u-v_{3} t}{v_{2}}\right)+\sum_{j=1}^{v_{4}} d_{j}\left\{\mathfrak{I}\left(a t-1,0,-\frac{v_{1}}{v_{2}}, \frac{u-g_{j} t}{v_{2}}\right)-\right.\right. \\
& \left.\left.e^{\frac{\lambda\left(g_{j} t-u\right)}{v_{3}-g_{j}}}\left[\Im\left(a t-1, h_{j}, c_{j}, \frac{u-g_{j} t}{v_{2}}\right)-\Im\left(a t-1, h_{j}, c_{j}, \frac{u-v_{3} t}{v_{2}}\right)\right]\right\} I_{\left\{g_{j}<v_{3}\right\}}\right),
\end{aligned}
$$


where

$$
\mathfrak{I}\left(\alpha, u_{1}, u_{2}, u_{3}\right)=\mathfrak{I}_{=}\left(\alpha, u_{2}, u_{3}\right)+\mathfrak{I}_{>}\left(\alpha, u_{1}, u_{2}, u_{3}\right)+\mathfrak{I}_{<}\left(\alpha, u_{1}, u_{2}, u_{3}\right)
$$

and $\mathfrak{I}_{=}, \mathfrak{I}_{>}, \mathfrak{I}_{<}$defined in (9), (10), (11), respectively. The next theorem gives us the distribution function of the random variable $X_{t}$.

Theorem 1. The distribution function

$$
F_{X_{t}}(u)=\mathfrak{V}\left(\theta, \sigma, \mu, m, \mathrm{p}_{j}, \mu_{j}, w_{j}, z_{j}, u ; j=1, \ldots, m\right) .
$$

The probability density function of $X_{t}$ is determined by the theorem below.

Theorem 2. The probability density function

$$
\begin{aligned}
f_{X_{t}}(u) & =\mathfrak{L}(0, u-\mu t)+\sum_{j=1}^{m} \mathrm{p}_{j}\left[\mathfrak{L}\left(0, u-\mu_{j} t\right)-\right. \\
-e^{\frac{\lambda\left(\mu_{j} t-u\right)}{\mu-\mu_{j}}} & \left.\left\{\mathfrak{L}\left(\frac{\lambda}{\mu-\mu_{j}}, u-\mu_{j} t\right)-\mathfrak{L}\left(\frac{\lambda}{\mu-\mu_{j}}, u-\mu t\right)\right\}\right] I_{\left\{\mu_{j}<\mu\right\}},
\end{aligned}
$$

where $\mathfrak{L}\left(u_{1}, u_{2}\right)$ is set by $(8)$.

Further, let

$$
\begin{aligned}
& \mathfrak{G}\left(v_{1}, v_{2}, v_{3}, v_{4}, v_{5}, d_{1 j}, d_{2 j}, d_{3 j}, d_{4 j}, d_{5 j}, d_{6 j}, d_{7 j}, d_{8 j}, u ; j=1, \ldots, v_{5}\right)= \\
& =\frac{a^{a t}}{\Gamma(a t)}\left(\Im\left(a t, 0,-\frac{v_{1}}{v_{2}}, \frac{u-v_{3} t}{v_{2}}\right)+v_{4} \mathfrak{I}\left(a t-1,0,-\frac{v_{1}}{v_{2}}, \frac{u-v_{3} t}{\sigma}\right)+\right. \\
& +\sum_{j=1}^{v_{5}} d_{1 j} I_{\left\{d_{2 j}<v_{3}\right.}\left\{\left\{d_{3 j} \Im\left(a t-1,0,-\frac{v_{1}}{v_{2}}, \frac{u-v_{3} t}{v_{2}}\right)+\right.\right. \\
& +d_{4 j} \mathfrak{I}\left(a t-1, d_{5 j}, d_{6 j}, \frac{u-v_{3} t}{v_{2}}\right)+d_{7 j} \Im\left(a t-1,0,-\frac{v_{1}}{v_{2}}, \frac{u-d_{2 j} t}{\sigma}\right)+ \\
& \left.\left.+\Im\left(a t, 0,-\frac{v_{1}}{v_{2}}, \frac{u-d_{2 j} t}{v_{2}}\right)+d_{8 j} \Im\left(a t-1, d_{5 j}, d_{6 j}, \frac{u-d_{2 j} t}{v_{2}}\right)\right\}\right)
\end{aligned}
$$

and set

$$
\begin{aligned}
& c=t\left(\mu\left[\mathrm{p}+e^{-\lambda t} \sum_{j=1}^{m} \mathrm{p}_{j}\right]+\left(1-e^{-\lambda t}\right) \sum_{j=1}^{m} \mathrm{p}_{j} \mu_{j}\right), \\
& c_{1 j}=\left(\mu_{j}-\mu\right) e^{-\lambda t}\left(t+\frac{1}{\lambda}\right)-t \mu_{j}, \\
& c_{2 j}=e^{\frac{\lambda\left(\mu_{j} t-u\right)}{\mu-\mu_{j}}}\left(u+\frac{\mu-\mu_{j}}{\lambda}\right), \\
& c_{3 j}=t \mu_{j}+\frac{\mu-\mu_{j}}{\lambda}, \\
& c_{4 j}=-e^{\frac{\lambda\left(\mu_{j} t-u\right)}{\mu-\mu_{j}}}\left(u+\frac{\mu-\mu_{j}}{\lambda}\right) .
\end{aligned}
$$


The next theorem determines the value of the lower partial expectation $\operatorname{LPE}_{X_{t}}(u)$ of $X_{t}$ which is defined as

$$
\operatorname{LPE}_{X_{t}}(u)=\mathrm{E}\left(X_{t} I_{\left\{X_{t} \leq u\right\}}\right) .
$$

Theorem 3. The lower partial expectation

$$
\begin{aligned}
& \operatorname{LPE}_{X_{t}}(u)= \\
= & \mathfrak{G}\left(\theta, \sigma, \mu, c, m, \mathrm{p}_{j}, \mu_{j}, c_{1 j}, c_{2 j}, w_{j}, z_{j}, c_{3 j}, c_{4 j}, u ; j=1, \ldots, m\right) .
\end{aligned}
$$

\section{Risk Measurement}

The aim of this section is to calculate in our model the values of the basic monetary risk measures. That is, to compute the value at risk and the expected shortfall for the returns which are modeled with the random variable $X_{t}$ defined in (6).

\subsection{Risk Measures}

The value at risk was prescribed to financial institutions for the estimation of the portfolio losses by the regulations Basel I and Basel II. It is defined as the low quantile of the distribution of the losses with minus sign. If the losses are modeled by a random variable $\zeta$, then the value at risk $\operatorname{VaR}_{\alpha}(\varsigma)$ is defined as

$$
\operatorname{VaR}_{\alpha}(\varsigma)=-u_{\alpha}, \quad \text { where } u_{\alpha}=\inf \left\{u \in \mathbb{R}: F_{\zeta}(u) \geq \alpha\right\} .
$$

An improvement of the value at risk, the expected shortfall monetary risk measure was recommended to banks by the Basel III regulation. In comparison to the value at risk, the expected shortfall takes into account the size of possible losses. The expected shortfall is defined as

$$
\mathrm{ES}_{\alpha}(\varsigma)=-\frac{1}{\alpha}\left[L_{\zeta}\left(u_{\alpha}\right)+u_{\alpha}\left(\alpha-F_{\zeta}\left(u_{\alpha}\right)\right)\right],
$$

where $L_{\zeta}(u), u \in \mathbb{R}$, is the lower partial expectation of $\varsigma$, that is

$$
L_{\zeta}(u)=\mathrm{E}\left(\varsigma I_{\{\zeta \leq u\}}\right) .
$$

If the distribution of $\zeta$ is continuous, the expected shortfall coincides with the conditional value at risk monetary measure which was primarily introduced in Rockafellar and Uryasev (2000).

The quantile $u_{\alpha}$ can be found as the solution of the equation

$$
F_{\zeta}(u)-\alpha=0
$$

with a one of the root-finding algorithms, see Brent (1973); Press et al. (2007); Stoer and Bulirsch (2002) for details. The computation of the distribution function of losses in analytical or semi-analytical forms in various models is given in Armenti et al. (2018); Drapeu et al. (2014); Ivanov (2018). Chun et al. (2012); Mafusalov and Uryasev (2016) estimate the value at risk and the expected shortfall using the Monte Carlo simulations. Nonparametric methods for the calculation of these two risk measures are presented in Cai and Wang (2008); Chen and Tang (2005); Scaillet (2005).

\subsection{Model and Results}

We assume that there are $n$ assets $A_{1}, \ldots, A_{n}$ in the investment portfolio whose dynamics is determined as

$$
A_{l, t}-A_{l, 0}=\int_{0}^{t} \mu_{l}(s) d s+\theta_{l} \gamma_{t}+\sigma_{l} B_{\gamma^{\prime}}^{l}
$$


where $\theta_{l} \in \mathbb{R}, \sigma_{l}>0, \gamma_{t}$ is the gamma process and $B_{l, s}, l=1,2, \ldots, n$ are the Brownian motions which are correlated with coefficients $\rho_{l j}$. It is suggested that the Brownian motions are independent with the gamma process. Similarly to Section 2 we assume that

$$
\mu_{l}(s)=\mu_{l} I_{\{\tau>s\}}+\widehat{\mu}_{l} I_{\{\tau \leq s\}}, \quad s \geq 0,
$$

where the random variable $\widehat{\mu}$ has a multinomial distribution with

$$
\begin{gathered}
\widehat{\mu}_{l} \in\left\{\mu_{l 1}, \mu_{l 2}, \ldots, \mu_{l m_{l}}, \mu_{l}\right\}, \quad \mu_{l 1} \leq \mu_{l 2} \leq \cdots \leq \mu_{l m_{l}} \leq \mu_{l}, \\
\mathrm{P}\left(\widehat{\mu}=\mu_{l j}\right)=\mathrm{p}_{l j} \geq 0, \quad \mathrm{P}\left(\widehat{\mu}=\mu_{l}\right)=\mathrm{p}_{l} \geq 0, \quad \mathrm{p}_{l}+\sum_{j=1}^{m_{l}} \mathrm{p}_{l j}=1
\end{gathered}
$$

for $l=0,1, \ldots, n$ and $\tau$ has an exponential distribution.

It is supposed also that two low risk assets are included in the portfolio. Namely, $A$ with the dynamics

$$
\begin{gathered}
A_{t}-A_{0}=\int_{0}^{t} r(s) d s, \\
r(s)=r I_{\{\tau>s\}}+\widehat{r} I_{\{\tau \leq s\}}, \quad s \geq 0, \\
\widehat{r} \in\left\{r_{1}, r_{2}, \ldots, r_{m_{r}}, r\right\}, \quad r_{1} \leq r_{2} \leq \cdots \leq r_{m_{r}} \leq r, \\
\mathrm{P}\left(\widehat{r}=r_{j}\right)=\mathrm{p}_{r j} \geq 0, \quad \mathrm{P}(\widehat{r}=r)=\mathrm{p}_{r} \geq 0, \quad \mathrm{p}_{r}+\sum_{j=1}^{m} \mathrm{p}_{r j}=1
\end{gathered}
$$

and $A_{0}$ with the variation

$$
A_{0, t}-A_{0,0}=\int_{0}^{t} \mu_{0}(s) d s+\theta_{0} \gamma_{t}
$$

where $\theta_{0} \in \mathbb{R}$.

To assess the risks of the portfolio, it is enough to discuss the process

$$
\begin{aligned}
\bar{X}_{t}=x \triangle A_{t}+\sum_{l=0}^{n} x_{l} \triangle A_{l, t} & =x \int_{0}^{t} r(s) d s+\sum_{l=0}^{n} x_{l} \int_{0}^{t} \mu_{l}(s) d s+ \\
& +\left(\sum_{l=0}^{n} x_{l} \theta_{l}\right) \gamma_{t}+\sum_{l=1}^{n} x_{l} \sigma_{l} B_{\gamma_{t}}^{l} .
\end{aligned}
$$

Set

$$
\bar{\theta}=\sum_{l=0}^{n} x_{l} \theta_{l}, \quad \bar{\mu}=x r+\sum_{l=0}^{n} x_{l} \mu_{l}, \quad \bar{\sigma}=\sqrt{\sum_{l, j=1}^{n} \rho_{l j} x_{l} x_{j} \sigma_{l} \sigma_{j}} .
$$

Then

$$
\begin{gathered}
\bar{X}_{t} \stackrel{\text { Law }}{=}\left(\bar{\mu} \tau+\left(x \widehat{r}+\sum_{l=0}^{n} x_{l} \widehat{\mu}_{l}\right)(t-\tau)\right) I_{\{\tau \leq t\}}+ \\
+\bar{\mu} t I_{\{\tau>t\}}+\bar{\theta} \gamma_{t}+\left(\sum_{l=1}^{n} x_{l} \sigma_{l} N^{l}\right) \sqrt{\gamma_{t}}
\end{gathered}
$$


where $N^{1}, \ldots, N^{n}$ are the standard normal random variables correlated with the same coefficients as the related Brownian motions. Since

$$
\sum_{l=1}^{n} x_{l} \sigma_{l} N^{l} \stackrel{\text { Law }}{=} \bar{\sigma} N,
$$

we have that

$$
\bar{X}_{t} \stackrel{\text { Law }}{=}(\bar{\mu} \tau+M(t-\tau)) I_{\{\tau \leq t\}}+\bar{\mu} t I_{\{\tau>t\}}+\bar{\theta} \gamma_{t}+\bar{\sigma} B_{\gamma_{t}}
$$

with

$$
M=x \widehat{r}+\sum_{l=0}^{n} x_{l} \widehat{\mu}_{l}
$$

Let $r_{0}=r, \mathrm{p}_{r 0}=\mathrm{p}_{r}$ and $\mu_{l 0}=\mu_{l}, \mathrm{p}_{l 0}=\mathrm{p}_{l}$ for $l=1, \ldots, n$. Set

$$
M_{j i_{0} i_{1} \ldots i_{n}}=x r_{j}+\sum_{l=0}^{n} x_{l} \mu_{l i_{l}}
$$

and

$$
\overline{\mathrm{p}}_{j i_{0} i_{1} \ldots i_{n}}=\mathrm{p}_{r j} \prod_{l=0}^{n} \mathrm{p}_{l i_{l}} .
$$

Then we have that

$$
M \in\left\{M_{j i_{0} i_{1} \ldots i_{n}}, j \in\left\{0,1, \ldots, m_{r}\right\}, i_{l} \in\left\{0,1, \ldots, m_{l}\right\}, l=1, \ldots, n\right\}
$$

and

$$
\mathrm{P}\left(M=M_{j i_{0} i_{1} \ldots i_{n}}\right)=\overline{\mathrm{p}}_{j i_{0} i_{1} \ldots i_{n}} .
$$

Next, let us dispose the values of $M$ in the increasing order

$$
\bar{M}_{1} \leq \bar{M}_{2} \leq \cdots \leq \bar{M}_{\left(m_{r}+1\right)\left(m_{0}+1\right)\left(m_{1}+1\right) \ldots\left(m_{n}+1\right)-1} \leq \bar{M}
$$

and set

$$
\begin{gathered}
\mathrm{P}\left(M=\bar{M}_{j}\right)=\overline{\mathrm{p}}_{j} \geq 0, \quad \mathrm{P}(M=\bar{M})=\overline{\mathrm{p}} \geq 0, \\
\overline{\mathrm{p}}+{ }^{\left(m_{r}+1\right)\left(m_{0}+1\right)\left(m_{j=1}+1\right) \ldots\left(m_{n}+1\right)-1} \overline{\mathrm{p}}_{j}=1 .
\end{gathered}
$$

Let

$$
\bar{K}=\left(m_{r}+1\right)\left(m_{0}+1\right)\left(m_{1}+1\right) \ldots\left(m_{n}+1\right)-1,
$$

similarly to (12) and (13)

$$
\begin{aligned}
& \bar{w}_{j}=\frac{\lambda}{\bar{M}-\bar{M}_{j}}\left(\bar{\theta}+\frac{\lambda \bar{\sigma}^{2}}{2\left(\bar{M}-\bar{M}_{j}\right)}\right), \\
& \bar{z}_{j}=\frac{\lambda \bar{\sigma}}{\bar{M}_{j}-\bar{M}}-\frac{\bar{\theta}}{\bar{\sigma}}
\end{aligned}
$$


and analogously to (23)-(27)

$$
\begin{aligned}
& \bar{c}=t\left(\bar{M}\left[\overline{\mathrm{p}}+e^{-\lambda t} \sum_{j=1}^{\bar{K}} \overline{\mathrm{p}}_{j}\right]+\left(1-e^{-\lambda t}\right) \sum_{j=1}^{\bar{K}} \overline{\mathrm{p}}_{j} \bar{M}_{j}\right), \\
& \bar{c}_{1 j}=\left(\bar{M}_{j}-\bar{M}\right) e^{-\lambda t}\left(t+\frac{1}{\lambda}\right)-t \bar{M}_{j}, \\
& \bar{c}_{2 j}=e^{\frac{\lambda\left(\bar{M}_{j}^{\left.t-u_{\alpha}\right)}\right.}{\bar{M}-\bar{M}_{j}}}\left(u_{\alpha}+\frac{\bar{M}-\bar{M}_{j}}{\lambda}\right), \\
& \bar{c}_{3 j}=t \bar{M}_{j}+\frac{\bar{M}-\bar{M}_{j}}{\lambda}, \\
& \bar{c}_{4 j}=-e^{\frac{\lambda\left(\bar{M}_{j} t-u_{\alpha}\right)}{\bar{M}_{-} \bar{M}_{j}}}\left(u_{\alpha}+\frac{\bar{M}-\bar{M}_{j}}{\lambda}\right) .
\end{aligned}
$$

The following proposition issues immediately from Theorems 1 and 3.

Corollary 1. The value at risk $\operatorname{VaR}_{\alpha}\left(\bar{X}_{t}\right)$ is determined as the solution $u_{\alpha}$ of the equation

$$
\mathfrak{V}\left(\bar{\theta}, \bar{\sigma}, \bar{M}, \bar{K}, \overline{\mathrm{p}}_{j}, \bar{M}_{j}, \bar{w}_{j}, \bar{z}_{j}, u ; j=1, \ldots, \bar{K}\right)-\alpha=0
$$

with minus sign. The expected shortfall $\mathrm{ES}_{\alpha}\left(\bar{X}_{t}\right)$ is calculated with respect to the formula

$$
\mathrm{ES}_{\alpha}\left(\bar{X}_{t}\right)=\mathfrak{G}\left(\bar{\theta}, \bar{\sigma}, \bar{M}, \bar{c}, \bar{K}, \overline{\mathrm{p}}_{j}, \bar{M}_{j}, \bar{c}_{1 j}, \bar{c}_{2 j}, \bar{w}_{j}, \bar{z}_{j}, \bar{c}_{3 j}, \bar{c}_{4 j}, u_{\alpha} ; j=1, \ldots, \bar{K}\right) .
$$

\section{Discussion}

The considered model extends the variance-gamma one under the assumption that the linear drift rate could drop down at some random time moment. From the risk measurement point of view, the formula for the distribution function of the underlying process allows us to compute the value at risk. The knowledge of the lower partial expectation permits the calculation of the expected shortfall. Various approximate computations can be made using the formula for the probability density function. Further investigations may include the calculation of risk-adjusted performance measures, downside and upside betas in the discussed model. To generalize the model, we can assume a smaller dependence between the gamma processes. Different types of the distribution of the jump time might be studied as well.

\section{Conclusions}

We have established the analytical formulas for the value at risk and the expected shortfall monetary risk measures in the model driven by the variance-gamma process with the probability of the linear drift rate negative jump. The time of the rate jump has been modeled by the exponential distribution. The size of the jump has been modeled by the multinomial distribution. The obtained results in particular can be considered as a step to the computation of various complicated risk characteristics in more general stochastic models. From the modeling point of view, taking into account the impact of the federal funds rate changes leads us to a model which distinguishes better the real financial market dynamics. It is expected that the new model can give better results for the value at risk backtesting, see Christoffersen and Pelletier (2004) for details. In general, the value at risk and the expected shortfall computation not only meets the requirements of regulators and the Basel Committee on Banking Supervision (see for example Ming Chen (2018)), but also allows us to construct more complex and economically sound models (see in particular Keçeci et al. (2016); Barone Adesi (2016)) and also to control the insurance market (see Ramsey and Goodwin (2019)). 


\section{Proofs}

Proof of Theorem 1. Since the probability

$$
\mathrm{P}\left(X_{t} \leq u\right)=\mathrm{E}\left(\mathrm{E}\left(I_{\left\{X_{t} \leq u\right\}} \mid Y_{t}\right)\right),
$$

where

$$
\Upsilon_{t}=\theta \gamma_{t}+\sigma B_{\gamma_{t}}
$$

we might start from the computation of the function

$$
\begin{aligned}
g\left(Y_{t}\right) & =\mathrm{E}\left(I_{\left\{X_{t} \leq u\right\}} \mid Y_{t}\right)=\mathrm{E}\left(I_{\left\{X_{t} \leq u, \tau \leq t\right\}} \mid Y_{t}\right)+\mathrm{E}\left(I_{\left\{X_{t} \leq u, \tau>t\right\}} \mid Y_{t}\right)= \\
& =\mathrm{E}\left(I_{\left\{\tau \leq t, \hat{u}(t-\tau) \leq u-\mu \tau-Y_{t}\right\}} \mid Y_{t}\right)+e^{-\lambda t} I_{\left\{Y_{t} \leq u-\mu t\right\}} .
\end{aligned}
$$

One can observe that the conditional expectation

$$
\begin{aligned}
& \mathrm{E}\left(I_{\left\{\tau \leq t, \widehat{\mu}(t-\tau) \leq u-\mu \tau-Y_{t}\right\}} \mid Y_{t}\right)=\mathrm{E}\left(I_{\left\{\tau \leq t, \widehat{\mu}(t-\tau) \leq u-\mu \tau-Y_{t}, \widehat{\mu}=\mu\right\}} \mid Y_{t}\right)+ \\
& +\sum_{j=1}^{m} \mathrm{E}\left(I_{\left\{\tau \leq t, \widehat{\mu}(t-\tau) \leq u-\mu \tau-Y_{t}, \widehat{u}=\mu_{j}\right\}} \mid Y_{t}\right)=\mathrm{p}\left(1-e^{-\lambda t}\right) I_{\left\{Y_{t} \leq u-\mu t\right\}}+ \\
& +\sum_{j=1}^{m} \mathrm{p}_{j} \mathrm{E}\left(I_{\left\{\tau \leq t, \mu_{j}(t-\tau) \leq u-\mu \tau-Y_{t}\right\}} \mid Y_{t}\right) .
\end{aligned}
$$

The conditional probability

$$
\begin{aligned}
& \mathrm{P}\left(\tau \leq t,\left(\mu-\mu_{j}\right) \tau \leq u-\mu_{j} t-Y_{t} \mid Y_{t}\right)= \\
= & \mathrm{P}\left(\tau \leq \min \left\{t, \frac{u-\mu_{j} t-Y_{t}}{\mu-\mu_{j}}\right\} \mid Y_{t}\right) I_{\left\{Y_{t} \leq u-\mu_{j} t, \mu_{j}<\mu\right\}}+ \\
+ & \left(1-e^{-\lambda t}\right) I_{\left\{Y_{t} \leq u-\mu t, \mu_{j}=\mu\right\}}=\left(1-e^{-\lambda t}\right) I_{\left\{Y_{t} \leq u-\mu t\right\}}+ \\
+ & \left(1-e^{\lambda \frac{Y_{t}+\mu_{j} t-u}{\mu-\mu_{j}}}\right) I_{\left\{u-\mu t<Y_{t} \leq u-\mu_{j} t, \mu_{j}<\mu\right\}} .
\end{aligned}
$$

Now we have from (31) and (32) that

$$
\begin{aligned}
& \mathrm{E}\left(I_{\left\{\tau<t, \widehat{\mu}(t-\tau) \leq u-\mu \tau-Y_{t}\right\}} \mid Y_{t}\right)=\left(1-e^{-\lambda t}\right) I_{\left\{Y_{t} \leq u-\mu t\right\}}+ \\
& +\sum_{j=1}^{m} \mathrm{p}_{j}\left[\left(1-e^{\lambda \frac{Y_{t}+\mu_{j} t-u}{\mu-\mu_{j}}}\right) I_{\left\{Y_{t} \leq u-\mu_{j} t\right\}}-\right. \\
& \left.-\left(1-e^{\lambda \frac{Y_{t}+\mu_{j} t-u}{\mu-\mu_{j}}}\right) I_{\left\{Y_{t} \leq u-\mu t\right\}}\right] I_{\left\{\mu_{j}<\mu\right\}}
\end{aligned}
$$

and therefore it follows from (30) and (33) that

$$
\begin{aligned}
g\left(Y_{t}\right) & =I_{\left\{Y_{t} \leq u-\mu t\right\}}+\sum_{j=1}^{m} \mathrm{p}_{j}\left[\left(1-e^{\lambda \frac{Y_{t}+\mu_{j} t-u}{\mu-\mu_{j}}}\right) I_{\left\{Y_{t} \leq u-\mu_{j} t\right\}}+\right. \\
& \left.+e^{\lambda \frac{Y_{t}+\mu_{j} t-u}{\mu-\mu_{j}}} I_{\left\{Y_{t} \leq u-\mu t\right\}}\right] I_{\left\{\mu_{j}<\mu\right\}} .
\end{aligned}
$$


Because of $\mathrm{P}\left(X_{t} \leq u\right)=\operatorname{Eg}\left(Y_{t}\right)$, we have that

$$
\begin{gathered}
\mathrm{P}\left(X_{t} \leq u\right)=\mathrm{P}\left(Y_{t} \leq u-\mu t\right)+\sum_{j=1}^{m} \mathrm{p}_{j}\left[\mathrm{P}\left(Y_{t} \leq u-\mu_{j} t\right)-\right. \\
\left.-e^{\frac{\lambda\left(\mu_{j} t-u\right)}{\mu-\mu_{j}}} \mathrm{E}^{\frac{\lambda Y_{t}}{\mu-\mu_{j}}} I_{\left\{Y_{t} \leq u-\mu_{j} t\right\}}+e^{\frac{\lambda\left(\mu_{j} t-u\right)}{\mu-\mu_{j}}} \mathrm{E}^{\frac{\lambda Y_{t}}{\mu-\mu_{j}}} I_{\left\{Y_{t} \leq u-\mu t\right\}}\right] I_{\left\{\mu_{j}<\mu\right\}} .
\end{gathered}
$$

Now it is required to compute the expectation

$$
V=V(A, D)=\mathrm{E} e^{A Y_{t}} I_{\left\{Y_{t} \leq D\right\}}
$$

for $A \in\left\{0, \frac{\lambda}{\mu-\mu_{j}}\right\}$ and $D \in \mathbb{R}$. Then

$$
\begin{aligned}
& \mathrm{P}\left(X_{t} \leq u\right)=V(0, u-\mu t)+\sum_{j=1}^{m} \mathrm{p}_{j}\left[V\left(0, u-\mu_{j} t\right)-\right. \\
& \left.-e^{\frac{\lambda\left(\mu_{j} t-u\right)}{\mu-\mu_{j}}} V\left(\frac{\lambda}{\mu-\mu_{j}}, u-\mu_{j} t\right)+e^{\frac{\lambda\left(\mu_{j} t-u\right)}{\mu-\mu_{j}}} V\left(\frac{\lambda}{\mu-\mu_{j}}, u-\mu t\right)\right] I_{\left\{\mu_{j}<\mu\right\}} .
\end{aligned}
$$

Set

$$
v\left(\gamma_{t}\right)=\mathrm{E}\left(e^{A Y_{t}} I_{\left\{Y_{t} \leq D\right\}} \mid \gamma_{t}\right)=\mathrm{E}\left(e^{A\left(\theta \gamma_{t}+\sigma B_{\gamma_{t}}\right)} I_{\left\{\theta \gamma_{t}+\sigma B_{\gamma_{t}} \leq D\right\}} \mid \gamma_{t}\right) .
$$

Then

$$
\begin{aligned}
& v\left(\gamma_{t}\right)=\int_{-\infty}^{D} \frac{1}{\sigma \sqrt{2 \pi \gamma_{t}}} e^{A x-\frac{\left(x-\theta \gamma_{t}\right)^{2}}{2 \sigma^{2} \gamma_{t}}} d x= \\
& =e^{\frac{A\left(A \sigma^{2}+2 \theta\right) \gamma_{t}}{2}} \int_{-\infty}^{D} \frac{1}{\sigma \sqrt{2 \pi \gamma_{t}}} e^{-\frac{\left(x-\gamma_{t}\left(A \sigma^{2}+\theta\right)\right)^{2}}{2 \sigma^{2} \gamma_{t}}} d x= \\
& =e^{\frac{A\left(A \sigma^{2}+2 \theta\right) \gamma_{t}}{2}} \Psi\left(\frac{D-\gamma_{t}\left(A \sigma^{2}+\theta\right)}{\sigma \sqrt{\gamma_{t}}}\right)= \\
& =\Psi\left(-\frac{\theta}{\sigma} \sqrt{\gamma_{t}}+\frac{D}{\sigma \sqrt{\gamma_{t}}}\right) I_{\{A=0\}}+ \\
& \left.+e^{\frac{\lambda}{\mu-\mu_{j}}\left(\frac{\lambda \sigma^{2}}{2\left(\mu-\mu_{j}\right)}+\theta\right) \gamma_{t}} \Psi\left(\left(\frac{\lambda \sigma}{\mu_{j}-\mu}-\frac{\theta}{\sigma}\right) \sqrt{\gamma_{t}}+\frac{D}{\sigma \sqrt{\gamma_{t}}}\right) I_{\left\{A=\frac{\lambda}{\mu-\mu_{j}}\right.}\right\}
\end{aligned}
$$

and since $V=\operatorname{Ev}\left(\gamma_{t}\right)$, we have from (35) and (36) that

$$
\begin{aligned}
& \mathrm{P}\left(X_{t} \leq u\right)=\mathrm{E}\left[\Psi\left(-\frac{\theta}{\sigma} \sqrt{\gamma_{t}}+\frac{u-\mu t}{\sigma \sqrt{\gamma_{t}}}\right)\right]+ \\
& +\sum_{j=1}^{m} \mathrm{p}_{j} I_{\left\{\mu_{j}<\mu\right\}}\left\{\mathrm{E}\left[\Psi\left(-\frac{\theta}{\sigma} \sqrt{\gamma_{t}}+\frac{u-\mu_{j} t}{\sigma \sqrt{\gamma_{t}}}\right)\right]-\right. \\
& -e^{\frac{\lambda\left(\mu_{j} t-u\right)}{\mu-\mu_{j}}} \mathrm{E}\left[e^{\frac{\lambda}{\mu-\mu_{j}}\left(\frac{\lambda \sigma^{2}}{2\left(\mu-\mu_{j}\right)}+\theta\right) \gamma_{t}} \Psi\left(\left(\frac{\lambda \sigma}{\mu_{j}-\mu}-\frac{\theta}{\sigma}\right) \sqrt{\gamma_{t}}+\frac{u-\mu_{j} t}{\sigma \sqrt{\gamma_{t}}}\right)\right]- \\
& \left.-e^{\frac{\lambda\left(\mu_{j} t-u\right)}{\mu-\mu_{j}}} \mathrm{E}\left[e^{\frac{\lambda}{\mu-\mu_{j}}\left(\frac{\lambda \sigma^{2}}{2\left(\mu-\mu_{j}\right)}+\theta\right) \gamma_{t}} \Psi\left(\left(\frac{\lambda \sigma}{\mu_{j}-\mu}-\frac{\theta}{\sigma}\right) \sqrt{\gamma_{t}}+\frac{u-\mu t}{\sigma \sqrt{\gamma_{t}}}\right)\right]\right\} .
\end{aligned}
$$


Now it is easy to see from (37) that we need to calculate the integral

$$
\mathfrak{I}=\mathfrak{I}(\alpha, b, h, p)=\int_{0}^{\infty} x^{\alpha} e^{-(a-b) x} \Psi\left(h \sqrt{x}+\frac{p}{\sqrt{x}}\right) d x
$$

for $\alpha>-1, a>0, p \in \mathbb{R}$ and the vector $(h, b) \in\left\{\left(-\frac{\theta}{\sigma}, 0\right),\left(z_{j}, w_{j}\right)\right\}$, where $j=1,2, . ., m$. Then

$$
\begin{aligned}
& \frac{\Gamma(a t) \mathrm{P}\left(X_{t} \leq u\right)}{b^{a t}}= \\
= & \mathfrak{I}\left(a t-1,0,-\frac{\theta}{\sigma}, \frac{u-\mu t}{\sigma}\right)+\sum_{j=1}^{m} \mathrm{p}_{j} I_{\left\{\mu_{j}<\mu\right\}}\left\{\mathfrak{I}\left(a t-1,0,-\frac{\theta}{\sigma}, \frac{u-\mu_{j} t}{\sigma}\right)\right. \\
- & \left.e^{\frac{\lambda\left(\mu_{j} t-u\right)}{\mu-\mu_{j}}}\left[\mathfrak{I}\left(a t-1, w_{j}, z_{j}, \frac{u-\mu_{j} t}{\sigma}\right)-\mathfrak{I}\left(a t-1, w_{j}, z_{j}, \frac{u-\mu t}{\sigma}\right)\right]\right\} .
\end{aligned}
$$

Further, we derive the value of $\mathfrak{I}=\mathfrak{I}(\alpha, b, h, p)$ with respect to relations between the parameters.

Case 1. $a=b$.

Case 1.1. $h \geq 0$. Then obviously $\mathfrak{I}=\infty$.

Case 1.2. $h<0, p=0$. We have that

$$
\begin{aligned}
\mathfrak{I}=\int_{0}^{\infty} x^{\alpha} \Psi(h \sqrt{x}) d x & =-\frac{h}{2(\alpha+1) \sqrt{2 \pi}} \int_{0}^{\infty} x^{\alpha+\frac{1}{2}} e^{-\frac{h^{2} x}{2}} d x= \\
& =\frac{2^{\alpha} \Gamma\left(\alpha+\frac{3}{2}\right)}{(\alpha+1)|h|^{2(\alpha+1)} \sqrt{\pi}} .
\end{aligned}
$$

Case 1.3. $h<0, p \neq 0$. Integrating by parts, one can obtain that

$$
\begin{aligned}
\mathfrak{I} & =-\frac{1}{\alpha+1} \int_{0}^{\infty} x^{\alpha+1} d \Psi\left(h \sqrt{x}+\frac{p}{\sqrt{x}}\right)= \\
& =\frac{1}{2(\alpha+1) \sqrt{2 \pi}} \int_{0}^{\infty}\left(p x^{\alpha-\frac{1}{2}}-h x^{\alpha+\frac{1}{2}}\right) e^{-\frac{(h x+p)^{2}}{2 x}} d x .
\end{aligned}
$$

Formula 3.471.9 from Gradshteyn and Ryzhik (2007) includes the identity

$$
\int_{0}^{\infty} x^{\vartheta_{1}-1} e^{-\frac{\vartheta_{2}}{x}-\vartheta_{3} x} d x=2\left(\frac{\vartheta_{2}}{\vartheta_{3}}\right)^{\frac{\vartheta_{1}}{2}} \mathrm{~K}_{\vartheta_{1}}\left(2 \sqrt{\vartheta_{2} \vartheta_{3}}\right),
$$

where $\vartheta_{1} \in \mathbb{R}, \vartheta_{2}>0$ and $\vartheta_{3}>0$. Using (40), we get that

$$
\begin{aligned}
\mathfrak{I} & =\frac{e^{-h p}}{(\alpha+1) \sqrt{2 \pi}}\left(\frac{p|p|^{\alpha+\frac{1}{2}}}{|h|^{\alpha+\frac{1}{2}}} \mathrm{~K}_{\alpha+\frac{1}{2}}(|p h|)-\frac{h|p|^{\alpha+\frac{3}{2}}}{|h|^{\alpha+\frac{3}{2}}} \mathrm{~K}_{\alpha+\frac{3}{2}}(|p h|)\right)= \\
& =\frac{e^{-h p}|p|^{\alpha+\frac{1}{2}}}{(\alpha+1) \sqrt{2 \pi}|h|^{\alpha+\frac{1}{2}}}\left(p \mathrm{~K}_{\alpha+\frac{1}{2}}(|p h|)+|p| \mathrm{K}_{\alpha+\frac{3}{2}}(|p h|)\right) .
\end{aligned}
$$

Case 2. $a \neq b, p=0$. Then

$$
\mathfrak{I}=\frac{1}{|a-b|^{\alpha+1}} \tilde{\mathfrak{I}}
$$

where $\tilde{h}=\frac{h}{\sqrt{|a-b|}}$ and $\tilde{\mathfrak{I}}=\int_{0}^{\infty} x^{\alpha} e^{-\operatorname{sign}(a-b) x} \Psi(\tilde{h} \sqrt{x}) d x$. 
Case 2.1. $a>b$. According to Case 2.2 at p. 208 of Ano and Ivanov (2016)

$$
\mathfrak{I}=\frac{\Gamma\left(\alpha+\frac{3}{2}\right)}{\sqrt{2 \pi}(a-b)^{\alpha+1}}\left(\frac{\mathrm{B}\left(\frac{1}{2}, \alpha+1\right)}{\sqrt{2}}+\frac{h}{\sqrt{a-b}} \mathrm{~F}\left(\alpha+\frac{3}{2}, \frac{1}{2}, \frac{3}{2} ; \frac{h^{2}}{2(b-a)}\right)\right) .
$$

Case 2.2. $a<b, \tilde{h}>-\sqrt{2}$. Then $\mathfrak{I}=\infty$ with respect to Case 2.1 at p. 208 of Ano and Ivanov (2016).

Case 2.3. $a<b, \tilde{h}<-\sqrt{2}$. We have that

$$
\begin{aligned}
\tilde{\mathfrak{I}} & =\int_{0}^{\infty} x^{\alpha} e^{x} \Psi(\tilde{h} \sqrt{x}) d x=\int_{0}^{\infty} x^{\alpha} e^{x}\left(\int_{-\infty}^{\tilde{h}} \frac{\sqrt{x}}{\sqrt{2 \pi}} e^{-\frac{v^{2} x}{2}} d v\right) d x= \\
& =\frac{1}{\sqrt{2 \pi}} \int_{-\infty}^{\tilde{h}}\left(\int_{0}^{\infty} x^{\alpha+\frac{1}{2}} e^{-\left(\frac{v^{2}}{2}-1\right) x} d x\right) d v .
\end{aligned}
$$

Using 3.382.4 from Gradshteyn and Ryzhik (2007), we get from (41) that

$$
\tilde{\mathfrak{I}}=\frac{\Gamma\left(\alpha+\frac{3}{2}\right)}{\sqrt{2 \pi}} \int_{-\infty}^{\tilde{h}}\left(\frac{v^{2}}{2}-1\right)^{-\alpha-\frac{3}{2}} d v .
$$

Set

$$
u=\left(\frac{v^{2}}{2}-1\right)^{-1}
$$

Then

$$
v=-\sqrt{\frac{2}{u}+2}, \quad d v=u^{-\frac{3}{2}}(2+2 u)^{-\frac{1}{2}} d u
$$

and hence

$$
\begin{aligned}
\tilde{\mathfrak{I}} & =\frac{\Gamma\left(\alpha+\frac{3}{2}\right)}{2 \sqrt{\pi}} \int_{0}^{\frac{2}{\bar{h}^{2}-2}} u^{\alpha}(1+u)^{-\frac{1}{2}} d u= \\
& =\frac{2^{\alpha} \Gamma\left(\alpha+\frac{3}{2}\right)}{(\alpha+1)\left(\tilde{h}^{2}-2\right)^{\alpha+1} \sqrt{\pi}} \mathrm{F}\left(\frac{1}{2}, \alpha+1, \alpha+2 ; \frac{2}{2-\tilde{h}^{2}}\right),
\end{aligned}
$$

where the last identity follows from 3.194.1 in Gradshteyn and Ryzhik (2007). Therefore

$$
\mathfrak{I}=\frac{2^{\alpha} \Gamma\left(\alpha+\frac{3}{2}\right)}{(\alpha+1)\left(h^{2}-2(b-a)\right)^{\alpha+1} \sqrt{\pi}} \mathrm{F}\left(\frac{1}{2}, \alpha+1, \alpha+2 ; \frac{2(b-a)}{2(b-a)-h^{2}}\right) .
$$

Case 3. $a \neq b, p \neq 0$. We have that

$$
\mathfrak{I}=\frac{1}{|a-b|^{\alpha+1}} \tilde{\mathfrak{I}}
$$

where

$$
\tilde{h}=\frac{h}{\sqrt{|a-b|}}, \quad \tilde{p}=p \sqrt{|a-b|}
$$

and

$$
\tilde{\mathfrak{I}}=\int_{0}^{\infty} x^{\alpha} e^{-\operatorname{sign}(a-b) x} \Psi\left(\tilde{h} \sqrt{x}+\frac{\tilde{p}}{\sqrt{x}}\right) d x .
$$


Case 3.1. $a>b$. With respect to (21) of Ano and Ivanov (2016),

$$
\begin{aligned}
& \mathfrak{I}=\frac{|s|^{\alpha+\frac{1}{2}} e^{s}(1+q)^{\alpha+1}}{\sqrt{2 \pi}(a-b)^{\alpha+1}}\left[\mathrm { B } ( \alpha + 1 , 1 ) \left(|s| \mathrm{K}_{\alpha+\frac{3}{2}}(|s|)+\right.\right. \\
& \left.+s \mathrm{~K}_{\alpha+\frac{1}{2}}(|s|)\right) \Phi\left(\alpha+1,-\alpha, \alpha+2 ; \frac{1+q}{2},-s(1+q)\right)- \\
& \left.(1+q) s \mathrm{~B}(\alpha+2,1) \mathrm{K}_{\alpha+\frac{1}{2}}(|s|) \Phi\left(\alpha+2,-\alpha, \alpha+3 ; \frac{1+q}{2},-s(1+q)\right)\right],
\end{aligned}
$$

where

$$
q=\frac{\tilde{h}}{\sqrt{\tilde{h}^{2}+2}} \quad \text { and } \quad s=\tilde{p} \sqrt{\tilde{h}^{2}+2} .
$$

Case 3.2. $a<b, \tilde{h}>-\sqrt{2}$. We conclude that $\tilde{\mathfrak{I}}=\infty$ by Case 3.1 at p. 210 of Ano and Ivanov (2016).

Case 3.3. $a<b, \tilde{h}<-\sqrt{2}$. Let $D(v)$ and $H(v), v \leq V \in \mathbb{R}$, be two differentiable functions with $D(V)=\tilde{p}$ and $H(V)=\tilde{h}$. Since

$$
\begin{aligned}
& \Psi\left(\frac{D(v)}{\sqrt{x}}+H(v) \sqrt{x}\right)=\int_{-\infty}^{v} \Psi_{u}^{\prime}\left(\frac{D(u)}{\sqrt{x}}+H(u) \sqrt{x}\right) d u= \\
= & \int_{-\infty}^{v} \Psi_{\frac{D(u)}{\sqrt{x}}+H(u) \sqrt{x}}^{\prime}\left(\frac{D(u)}{\sqrt{x}}+H(u) \sqrt{x}\right)\left(\frac{D_{u}^{\prime}(u)}{\sqrt{x}}+H_{u}^{\prime}(u) \sqrt{x}\right) d u,
\end{aligned}
$$

one gets that

$$
\begin{aligned}
& \Psi\left(\frac{\tilde{p}}{\sqrt{x}}+\tilde{h} \sqrt{x}\right)= \\
& =\int_{-\infty}^{V} \frac{1}{\sqrt{2 \pi}} e^{-D(v) H(v)-\frac{D^{2}(v)}{2 x}-\frac{H^{2}(v) x}{2}}\left(\frac{D_{v}^{\prime}(v)}{\sqrt{x}}+H_{v}^{\prime}(v) \sqrt{x}\right) d v
\end{aligned}
$$

Set $V=\tilde{h}$ and

$$
H(v)=v, \quad D(v)=\frac{\tilde{p} \sqrt{\tilde{h}^{2}-2}}{\sqrt{v^{2}-2}}, \quad v \leq \tilde{h} .
$$

Then

$$
\begin{aligned}
\tilde{\mathfrak{I}} & =\frac{1}{\sqrt{2 \pi}} \int_{0}^{\infty} x^{\alpha} e^{x}\left[\int_{-\infty}^{\tilde{h}} e^{-\frac{\tilde{p} v \sqrt{\tilde{h}^{2}-2}}{\sqrt{v^{2}-2}}-\frac{\tilde{p}^{2}\left(\tilde{h}^{2}-2\right)}{2\left(v^{2}-2\right) x}-\frac{v^{2} x}{2}} \times\right. \\
& \left.\times\left(\sqrt{x}-\frac{\tilde{p} v \sqrt{\tilde{h}^{2}-2}}{\left(v^{2}-2\right)^{\frac{3}{2}} \sqrt{x}}\right) d v\right] d x .
\end{aligned}
$$

Let us consider the double integrals

$$
J_{1}=\int_{0}^{\infty} \int_{-\infty}^{\tilde{h}}\left(x^{\alpha+\frac{1}{2}} e^{x-\frac{\tilde{p} v \sqrt{\tilde{h}^{2}-2}}{\sqrt{v^{2}-2}}-\frac{\tilde{p}^{2}\left(\tilde{h}^{2}-2\right)}{2\left(v^{2}-2\right) x}-\frac{v^{2} x}{2}}\right) d v d x
$$

and

$$
J_{2}=\int_{0}^{\infty} \int_{-\infty}^{\tilde{h}}\left(\frac{\tilde{p} x^{\alpha-\frac{1}{2}} v \sqrt{\tilde{h}^{2}-2}}{\left(v^{2}-2\right)^{\frac{3}{2}}} e^{x-\frac{\tilde{p} v \sqrt{\tilde{h}^{2}-2}}{\sqrt{v^{2}-2}}-\frac{\tilde{p}^{2}\left(\tilde{h}^{2}-2\right)}{2\left(v^{2}-2\right) x}-\frac{v^{2} x}{2}}\right) d v d x
$$


One can notice that both $J_{1}$ and $J_{2}$ are integrals of constant sign functions. Therefore, if the iterated integrals

$$
\widehat{J}_{1}=\int_{-\infty}^{\tilde{h}}\left(\int_{0}^{\infty} x^{\alpha+\frac{1}{2}} e^{x-\frac{\tilde{p} v \sqrt{\hat{h}^{2}-2}}{\sqrt{v^{2}-2}}-\frac{\tilde{p}^{2}\left(\tilde{h}^{2}-2\right)}{2\left(v^{2}-2\right) x}-\frac{v^{2} x}{2}} d x\right) d v
$$

and

$$
\widehat{J}_{2}=\int_{-\infty}^{\tilde{h}}\left(\int_{0}^{\infty} \frac{\tilde{p} x^{\alpha-\frac{1}{2}} v \sqrt{\tilde{h}^{2}-2}}{\left(v^{2}-2\right)^{\frac{3}{2}}} e^{x-\frac{\tilde{p} v \sqrt{\tilde{h}^{2}-2}}{\sqrt{v^{2}-2}}-\frac{\tilde{p}^{2}\left(\tilde{h}^{2}-2\right)}{2\left(v^{2}-2\right) x}-\frac{v^{2} x}{2}} d x\right) d v
$$

exist, then $J_{1}=\widehat{J}_{1}, J_{2}=\widehat{J}_{2}$ and the Fubini's theorem can be exploited to $J_{1}$ and $J_{2}$, that is

$$
\tilde{\mathfrak{I}}=\frac{1}{\sqrt{2 \pi}}\left(\widehat{J}_{1}-\widehat{J}_{2}\right) .
$$

Set $s=\tilde{p} \sqrt{\tilde{h}^{2}-2}$. Then we have according to 3.471.9 from Gradshteyn and Ryzhik (2007) that

$$
\widehat{J}_{1}=2|s|^{\alpha+\frac{3}{2}} \mathrm{~K}_{\alpha+\frac{3}{2}}(|s|) \int_{-\infty}^{\tilde{h}}\left(v^{2}-2\right)^{-\alpha-\frac{3}{2}} e^{-\frac{s v}{\sqrt{v^{2}-2}}} d v
$$

and

$$
\widehat{J}_{2}=2 s|s|^{\alpha+\frac{1}{2}} \mathrm{~K}_{\alpha+\frac{1}{2}}(|s|) \int_{-\infty}^{\tilde{h}} v\left(v^{2}-2\right)^{-\alpha-2} e^{-\frac{s v}{\sqrt{v^{2}-2}}} d v .
$$

Next, let us make the change of variables

$$
v \rightarrow y, \quad y=-\frac{v}{\sqrt{v^{2}-2}} .
$$

Then

$$
v=-\frac{y \sqrt{2}}{\sqrt{y^{2}-1}}, \quad v^{2}-2=\frac{2}{y^{2}-1}, \quad d v=\frac{\sqrt{2}}{\left(y^{2}-1\right)^{\frac{3}{2}}} d y
$$

and one can get that

$$
\widehat{J}_{1}=2^{-\alpha}|s|^{\alpha+\frac{3}{2}} K_{\alpha+\frac{3}{2}}(|s|) \int_{1}^{\frac{|\tilde{h}|}{\sqrt{\tilde{h}^{2}-2}}}\left(y^{2}-1\right)^{\alpha} e^{s y} d y
$$

and

$$
\widehat{J}_{2}=-2^{-\alpha} s|s|^{\alpha+\frac{1}{2}} K_{\alpha+\frac{1}{2}}(|s|) \int_{1}^{\frac{|\tilde{h}|}{\sqrt{\tilde{h}^{2}-2}}} y\left(y^{2}-1\right)^{\alpha} e^{s y} d y .
$$

Set

$$
q=\frac{|\tilde{h}|}{\sqrt{\tilde{h}^{2}-2}} \quad \text { and } \quad u=\frac{y-1}{q-1}
$$

Then

$$
\begin{aligned}
\widehat{J}_{1} & =2^{-\alpha}(q-1)|s|^{\alpha+\frac{3}{2}} e^{s} \mathrm{~K}_{\alpha+\frac{3}{2}}(|s|) \int_{0}^{1}\left((q-1)^{2} u^{2}+2(q-1) u\right)^{\alpha} e^{s(q-1) u} d u \\
& =(q-1)^{\alpha+1}|s|^{\alpha+\frac{3}{2}} e^{s} K_{\alpha+\frac{3}{2}}(|s|) \int_{0}^{1} u^{\alpha}\left(\frac{(q-1) u}{2}+1\right)^{\alpha} e^{s(q-1) u} d u
\end{aligned}
$$


and

$$
\begin{aligned}
& \widehat{J}_{2}= \\
& -2^{-\alpha}(q-1) s|s|^{\alpha+\frac{1}{2}} e^{s} \mathrm{~K}_{\alpha+\frac{1}{2}}(|s|)\left(\int_{0}^{1}\left((q-1)^{2} u^{2}+2(q-1) u\right)^{\alpha} e^{s(q-1) u} d u\right. \\
& \left.+(q-1) \int_{0}^{1} u\left((q-1)^{2} u^{2}+2(q-1) u\right)^{\alpha} e^{s(q-1) u} d u\right)= \\
& =-(q-1)^{\alpha+1} s|s|^{\alpha+\frac{1}{2}} e^{s} \mathrm{~K}_{\alpha+\frac{1}{2}}(|s|)\left(\int_{0}^{1} u^{\alpha}\left(\frac{(q-1) u}{2}+1\right)^{\alpha} e^{s(q-1) u} d u+\right. \\
& \left.+(q-1) \int_{0}^{1} u^{\alpha+1}\left(\frac{(q-1) u}{2}+1\right)^{\alpha} e^{s(q-1) u} d u\right) .
\end{aligned}
$$

Applying 3.385 of Gradshteyn and Ryzhik (2007) to (44) and (45) and keeping in mind (43), we establish now that

$$
\begin{aligned}
\mathfrak{I} & =\frac{(q-1)^{\alpha+1}|s|^{\alpha+\frac{1}{2}} e^{s}}{(b-a)^{\alpha+1} \sqrt{2 \pi}}\left[\mathrm{B}(\alpha+1,1) \Phi\left(\alpha+1,-\alpha, \alpha+2 ; \frac{1-q}{2}, s(1-q)\right) \times\right. \\
& \times\left(|s| \mathrm{K}_{\alpha+\frac{3}{2}}(|s|)+s \mathrm{~K}_{\alpha+\frac{1}{2}}(|s|)\right)+(q-1) s \mathrm{~K}_{\alpha+\frac{1}{2}}(|s|) \mathrm{B}(\alpha+2,1) \times \\
& \left.\times \Phi\left(\alpha+2,-\alpha, \alpha+3 ; \frac{1-q}{2}, s(1-q)\right)\right] .
\end{aligned}
$$

Now we have from Cases 1 to 3 that

$$
\begin{aligned}
& \Im\left(\alpha, 0,-\frac{\theta}{\sigma}, \frac{u-\bar{\mu} t}{\sigma}\right)= \\
= & \frac{\Gamma\left(\alpha+\frac{3}{2}\right)}{a^{\alpha+1} \sqrt{2 \pi}}\left(\frac{\mathrm{B}\left(\frac{1}{2}, \alpha+1\right)}{\sqrt{2}}-\frac{\theta}{\sigma \sqrt{a}} \mathrm{~F}\left(\alpha+\frac{3}{2}, \frac{1}{2}, \frac{3}{2} ;-\frac{\theta^{2}}{2 \sigma^{2} a}\right)\right) I_{\{u=\bar{\mu} t\}}+ \\
+ & \frac{|s|^{\alpha+\frac{1}{2}} e^{s}(1+q)^{\alpha+1}}{a^{\alpha+1} \sqrt{2 \pi}}\left[\mathrm{B}(\alpha+1,1)\left(|s| \mathrm{K}_{\alpha+\frac{3}{2}}(|s|)+s \mathrm{~K}_{\alpha+\frac{1}{2}}(|s|)\right) \times\right. \\
\times & \Phi\left(\alpha+1,-\alpha, \alpha+2 ; \frac{1+q}{2},-s(1+q)\right)-(1+q) s \mathrm{~B}(\alpha+2,1) \mathrm{K}_{\alpha+\frac{1}{2}}(|s|) \times \\
\times & \left.\Phi\left(\alpha+2,-\alpha, \alpha+3 ; \frac{1+q}{2},-s(1+q)\right)\right] I_{\{u \neq \bar{\mu} t\}},
\end{aligned}
$$

with

$$
q=-\frac{\theta}{\sqrt{\theta^{2}+2 \sigma^{2} a}} \quad \text { and } \quad s=\frac{(u-\bar{\mu} t) \sqrt{\theta^{2}+2 \sigma^{2} a}}{\sigma^{2}}
$$

where $\bar{\mu} \in\left\{\mu_{j}, \mu\right\}, j=1,2, \ldots, m$.

Further, since if $w_{j}>0$, then $z_{j}<0$ and

$$
\frac{z_{j}^{2}}{w_{j}}=\frac{\frac{\theta^{2}}{\sigma^{2}}+\frac{2 \lambda \theta}{\mu-\mu_{j}}+\frac{\lambda^{2} \sigma^{2}}{\left(\mu-\mu_{j}\right)^{2}}}{\frac{\lambda \theta}{\mu-\mu_{j}}+\frac{\lambda^{2} \sigma^{2}}{2\left(\mu-\mu_{j}\right)^{2}}} \geq 2,
$$


we have that

$$
\begin{aligned}
& \mathfrak{I}\left(\alpha, w_{j}, z_{j}, \frac{u-\bar{\mu} t}{\sigma}\right)= \\
= & \mathfrak{I}_{=}\left(\alpha, w_{j}, z_{j}, \frac{u-\bar{\mu} t}{\sigma}\right)+\mathfrak{I}_{>}\left(\alpha, w_{j}, z_{j}, \frac{u-\bar{\mu} t}{\sigma}\right)+\mathfrak{I}_{<}\left(\alpha, w_{j}, z_{j}, \frac{u-\bar{\mu} t}{\sigma}\right)
\end{aligned}
$$

with

$$
\begin{aligned}
& \mathfrak{I}_{=}\left(\alpha, w_{j}, z_{j}, \frac{u-\bar{\mu} t}{\sigma}\right)=\frac{2^{\alpha} \Gamma\left(\alpha+\frac{3}{2}\right)}{(\alpha+1)\left|z_{j}\right|^{2(\alpha+1)} \sqrt{\pi}} I_{\left\{a=w_{j}, u=\bar{\mu} t\right.}+ \\
& +\frac{e^{\frac{z_{j}(u-\bar{\mu} t)}{\sigma}}|u-\bar{\mu} t|^{\alpha+\frac{3}{2}}}{(\alpha+1) \sqrt{2 \pi}\left|z_{j}\right|^{\alpha+\frac{1}{2}} \sigma^{\alpha+\frac{3}{2}}}\left[\operatorname{sgn}(u-\bar{\mu} t) \mathrm{K}_{\alpha+\frac{1}{2}}\left(\left|\frac{z_{j}(u-\bar{\mu} t)}{\sigma}\right|\right)+\right. \\
& \left.+\mathrm{K}_{\alpha+\frac{3}{2}}\left(\left|\frac{z_{j}(u-\bar{\mu} t)}{\sigma}\right|\right)\right] I_{\left\{a=w_{j}, u \neq \bar{\mu} t\right\}} \\
& \mathfrak{I}_{>}\left(\alpha, w_{j}, z_{j}, \frac{u-\bar{\mu} t}{\sigma}\right)=\frac{\Gamma\left(\alpha+\frac{3}{2}\right)}{\sqrt{2 \pi}\left(a-w_{j}\right)^{\alpha+1}}\left[\frac{\mathrm{B}\left(\frac{1}{2}, \alpha+1\right)}{\sqrt{2}}+\right. \\
& \left.+\frac{z_{j}}{\sqrt{a-w_{j}}} \mathrm{~F}\left(\alpha+\frac{3}{2}, \frac{1}{2}, \frac{3}{2} ; \frac{z_{j}^{2}}{2\left(w_{j}-a\right)}\right)\right] I_{\left\{a>w_{j}, u=\bar{\mu} t\right.}+ \\
& +\frac{\left|s_{>}\right|^{\alpha+\frac{1}{2}} e^{s_{>}}(1+q>)^{\alpha+1}}{\sqrt{2 \pi}\left(a-w_{j}\right)^{\alpha+1}}\left[\mathrm{~B}(\alpha+1,1)\left(\left|s_{>}\right| \mathrm{K}_{\alpha+\frac{3}{2}}\left(\left|s_{>}\right|\right)+s_{>} \mathrm{K}_{\alpha+\frac{1}{2}}\left(\left|s_{>}\right|\right)\right)\right. \\
& \times \Phi\left(\alpha+1,-\alpha, \alpha+2 ; \frac{1+q_{>}}{2},-s_{>}\left(1+q_{>}\right)\right)-\left(1+q_{>}\right) s_{>} \mathrm{B}(\alpha+2,1) \times \\
& \left.\times \mathrm{K}_{\alpha+\frac{1}{2}}\left(\left|s_{>}\right|\right) \Phi\left(\alpha+2,-\alpha, \alpha+3 ; \frac{1+q_{>}}{2},-s_{>}\left(1+q_{>}\right)\right)\right] I_{\left\{a>w_{j}, u \neq \bar{\mu} t\right.}, \\
& q_{>}=\frac{z_{j}}{\sqrt{z_{j}^{2}+2\left(a-w_{j}\right)}}, \quad s_{>}=\frac{(u-\bar{\mu} t) \sqrt{z_{j}^{2}+2\left(a-w_{j}\right)}}{\sigma} \\
& \mathfrak{I}_{<}\left(\alpha, w_{j}, z_{j}, \frac{u-\bar{\mu} t}{\sigma}\right)=\frac{2^{\alpha} \Gamma\left(\alpha+\frac{3}{2}\right)}{(\alpha+1)\left(z_{j}^{2}-2\left(w_{j}-a\right)\right)^{\alpha+1} \sqrt{\pi}} \times \\
& \times \mathrm{F}\left(\frac{1}{2}, \alpha+1, \alpha+2 ; \frac{2\left(w_{j}-a\right)}{2\left(w_{j}-a\right)-z_{j}^{2}}\right) I_{\left\{a<w_{j}, u=\bar{\mu} t\right\}}+ \\
& \frac{\left(q_{<}-1\right)^{\alpha+1}\left|s_{<}\right|^{\alpha+\frac{1}{2}} e^{s_{<}}}{\left(w_{j}-a\right)^{\alpha+1} \sqrt{2 \pi}}\left[\mathrm{B}(\alpha+1,1) \Phi\left(\alpha+1,-\alpha, \alpha+2 ; \frac{1-q_{<}}{2}, s_{<}\left(1-q_{<}\right)\right)\right. \\
& \times\left(\left|s_{<}\right| \mathrm{K}_{\alpha+\frac{3}{2}}\left(\left|s_{<}\right|\right)+s_{<} \mathrm{K}_{\alpha+\frac{1}{2}}\left(\left|s_{<}\right|\right)\right)+(q<-1) s_{<} \mathrm{K}_{\alpha+\frac{1}{2}}\left(\left|s_{<}\right|\right) \mathrm{B}(\alpha+2,1) \times \\
& \left.\left.\times \Phi\left(\alpha+2,-\alpha, \alpha+3 ; \frac{1-q_{<}}{2}, s_{<}(1-q<)\right)\right] I_{\left\{a<w_{j}, u \neq \bar{\mu} t\right.}\right\}^{\prime}
\end{aligned}
$$




$$
q_{<}=-\frac{z_{j}}{\sqrt{z_{j}^{2}-2\left(w_{j}-a\right)}}, \quad s_{<}=\frac{(u-\bar{\mu} t) \sqrt{z_{j}^{2}-2\left(w_{j}-a\right)}}{\sigma},
$$

where $\bar{\mu} \in\left\{\mu_{j}, \mu\right\}, j=1,2, \ldots, m$.

Finally, we get (20) from (19), (39), (46) and (47).

Proof of Theorem 2. With respect to (35), the distribution function

$$
\begin{gathered}
F_{X_{t}}(u)=\mathrm{P}\left(X_{t} \leq u\right)=V(0, u-\mu t)+\sum_{j=1}^{m} \mathrm{p}_{j}\left[V\left(0, u-\mu_{j} t\right)-\right. \\
\left.-e^{\frac{\lambda\left(\mu_{j} t-u\right)}{\mu-\mu_{j}}} V\left(\frac{\lambda}{\mu-\mu_{j}}, u-\mu_{j} t\right)+e^{\frac{\lambda\left(\mu_{j} t-u\right)}{\mu-\mu_{j}}} V\left(\frac{\lambda}{\mu-\mu_{j}}, u-\mu t\right)\right] I_{\left\{\mu_{j}<\mu\right\}},
\end{gathered}
$$

where $V(A, D)=\operatorname{Ev}\left(\gamma_{t} ; A, D\right)$ with

$$
\begin{aligned}
& v\left(\gamma_{t} ; A, D\right)=\Psi\left(-\frac{\theta}{\sigma} \sqrt{\gamma_{t}}+\frac{D}{\sigma \sqrt{\gamma_{t}}}\right) I_{\{A=0\}}+ \\
& +e^{\frac{\lambda}{\mu-\mu_{j}}\left(\frac{\lambda \sigma^{2}}{2\left(\mu-\mu_{j}\right)}+\theta\right) \gamma_{t}} \Psi\left(\left(\frac{\lambda \sigma}{\mu_{j}-\mu}-\frac{\theta}{\sigma}\right) \sqrt{\gamma_{t}}+\frac{D}{\sigma \sqrt{\gamma_{t}}}\right) I_{\left\{A=\frac{\lambda}{\mu-\mu_{j}}\right\}^{\prime}}
\end{aligned}
$$

see (36). We have for $A \in\left\{0, \frac{\lambda}{\mu-\mu_{j}}\right\}$ that

$$
\begin{aligned}
& V_{u}^{\prime}(A, u-\bar{\mu} t)=\mathrm{Ev}_{u}^{\prime}\left(\gamma_{t} ; A, u-\bar{\mu} t\right)=\frac{1}{\sigma \sqrt{2 \pi}} \mathrm{E} \gamma_{t}^{-\frac{1}{2}} e^{-\frac{\left(u-\bar{\mu} t-\theta \gamma_{t}\right)^{2}}{2 \sigma^{2} \gamma_{t}}} I_{\{A=0\}} \\
& +\frac{1}{\sigma \sqrt{2 \pi}} \mathrm{E} \gamma_{t}^{-\frac{1}{2}} e^{\frac{\lambda}{\mu-\mu_{j}}\left(\frac{\lambda \sigma^{2}}{2\left(\mu-\mu_{j}\right)}+\theta\right) \gamma_{t}-\frac{\left(u-\bar{\mu} t-\left(\theta+\lambda \sigma^{2} /\left(\mu-\mu_{j}\right)\right) \gamma_{t}\right)^{2}}{2 \sigma^{2} \gamma_{t}}} I_{\left\{A=\frac{\lambda}{\mu-\mu_{j}}\right\}} .
\end{aligned}
$$

Since

$$
\int_{0}^{\infty} x^{\chi_{1}-1} e^{-\frac{\chi_{2}}{x}-\chi_{3} x} d x=2\left(\frac{\chi_{2}}{\chi_{3}}\right)^{\frac{\chi_{1}}{2}} \mathrm{~K}_{\chi_{1}}\left(2 \sqrt{\chi_{2} \chi_{3}}\right)
$$

for $\chi_{1} \in \mathbb{R}, \chi_{2}>0, \chi_{3}>0$ with respect to formula 3.471.9 from Gradshteyn and Ryzhik (2007), we get for $u \neq \bar{\mu} t$ that the expectations

$$
\begin{aligned}
& \mathrm{E} \gamma_{t}^{-\frac{1}{2}} e^{-\frac{\left(u-\bar{\mu} t-\theta \gamma_{t}\right)^{2}}{2 \sigma^{2} \gamma_{t}}}=\frac{a^{a t} e^{\frac{\theta(u-\bar{\mu} t)}{\sigma^{2}}}}{\Gamma(a t)} \int_{0}^{\infty} x^{a t-\frac{3}{2}} e^{-\frac{(u-\overline{-} t)^{2}}{2 \sigma^{2} x}-\left(a+\frac{\theta^{2}}{2 \sigma^{2}}\right) x} d x= \\
& =\frac{2 a^{a t} e^{\frac{\theta(u-\bar{\mu} t)}{\sigma^{2}}}}{\Gamma(a t)}\left(\frac{|u-\bar{\mu} t|}{\sqrt{2 a \sigma^{2}+\theta^{2}}}\right)^{a t-\frac{1}{2}} \mathrm{~K}_{a t-\frac{1}{2}}\left(\frac{|u-\bar{\mu} t| \sqrt{2 a \sigma^{2}+\theta^{2}}}{\sigma^{2}}\right)
\end{aligned}
$$

and

$$
\begin{aligned}
& \mathrm{E}_{t}^{-\frac{1}{2}} e^{\frac{\lambda}{\mu-\mu_{j}}\left(\frac{\lambda \sigma^{2}}{2\left(\mu-\mu_{j}\right)}+\theta\right) \gamma_{t}-\frac{\left(u-\bar{\mu} t-\left(\theta+\lambda \sigma^{2} /\left(\mu-\mu_{j}\right)\right) \gamma_{t}\right)^{2}}{2 \sigma^{2} \gamma_{t}}}= \\
& \frac{a^{a t} e^{\frac{\left(\theta+\lambda \sigma^{2} /\left(\mu-\mu_{j}\right)\right)(u-\bar{\mu} t)}{\sigma^{2}}}}{\Gamma(a t)} \int_{0}^{\infty} x^{a t-\frac{3}{2}} e^{-\frac{(u-\bar{\mu} t)^{2}}{2 \sigma^{2} x}-\left(a+\frac{\theta^{2}}{2 \sigma^{2}}\right) x} d x= \\
& \frac{2 a^{a t} e^{\frac{\left(\theta+\lambda \sigma^{2} /\left(\mu-\mu_{j}\right)\right)(u-\bar{\mu} t)}{\sigma^{2}}}}{\Gamma(a t)}\left(\frac{|u-\bar{\mu} t|}{\sqrt{2 a \sigma^{2}+\theta^{2}}}\right)^{a t-\frac{1}{2}} \mathrm{~K}_{a t-\frac{1}{2}}\left(\frac{|u-\bar{\mu} t| \sqrt{2 a \sigma^{2}+\theta^{2}}}{\sigma^{2}}\right) .
\end{aligned}
$$


Now we have from (48)-(51) that if $u \notin\left\{\mu_{1} t, \mu_{2} t, \ldots, \mu_{m} t, \mu t\right\}$, then the probability density function

$$
\begin{gathered}
f_{X_{t}}(u)=F_{X_{t}}^{\prime}(u)=\tilde{\mathfrak{L}}(0, u-\mu t)+\sum_{j=1}^{m} \mathrm{p}_{j}\left[\tilde{\mathfrak{L}}\left(0, u-\mu_{j} t\right)-\right. \\
\left.-e^{\frac{\lambda\left(\mu_{j} t-u\right)}{\mu-\mu_{j}}} \tilde{\mathfrak{L}}\left(\frac{\lambda}{\mu-\mu_{j}}, u-\mu_{j} t\right)+e^{\frac{\lambda\left(\mu_{j} t-u\right)}{\mu-\mu_{j}}} \tilde{\mathfrak{L}}\left(\frac{\lambda}{\mu-\mu_{j}}, u-\mu t\right)\right] I_{\left\{\mu_{j}<\mu\right\}},
\end{gathered}
$$

where

$$
\begin{aligned}
& \tilde{\mathfrak{L}}\left(u_{1}, u_{2}\right)= \\
= & \frac{2 a^{a t} e^{\frac{\theta u_{2}}{\sigma^{2}}}}{\sigma \Gamma(a t) \sqrt{2 \pi}}\left(\frac{\left|u_{2}\right|}{\sqrt{2 a \sigma^{2}+\theta^{2}}}\right)^{a t-\frac{1}{2}} \mathrm{~K}_{a t-\frac{1}{2}}\left(\frac{\left|u_{2}\right| \sqrt{2 a \sigma^{2}+\theta^{2}}}{\sigma^{2}}\right) I_{\left\{u_{1}=0\right\}}+ \\
+ & \frac{2 a^{a t} e^{\frac{\left(\theta+u_{1} \sigma^{2}\right) u_{2}}{\sigma^{2}}}}{\sigma \Gamma(a t) \sqrt{2 \pi}}\left(\frac{\left|u_{2}\right|}{\sqrt{2 a \sigma^{2}+\theta^{2}}}\right)^{a t-\frac{1}{2}} \mathrm{~K}_{a t-\frac{1}{2}}\left(\frac{\left|u_{2}\right| \sqrt{2 a \sigma^{2}+\theta^{2}}}{\sigma^{2}}\right) I_{\left\{u_{1} \neq 0\right\}}
\end{aligned}
$$

for $u_{2} \neq 0$.

Send $u_{2} \rightarrow 0$ in (53). Using the asymptotics

$$
\mathrm{K}_{\chi_{1}}\left(\chi_{2}\right) \sim \frac{\Gamma\left(\chi_{1}\right)}{2}\left(\frac{2}{\chi_{2}}\right)^{\chi_{1}}, \chi_{1}>0, \text { as } \chi_{2} \rightarrow 0,
$$

we get that

$$
\begin{aligned}
& \quad \tilde{\mathfrak{L}}\left(u_{1}, u_{2}\right) \sim \\
& \sim \frac{a^{a t} \Gamma\left(a t-\frac{1}{2}\right)}{\sigma\left(a+\frac{\theta^{2}}{2 \sigma^{2}}\right)^{a t-\frac{1}{2}} \Gamma(a t) \sqrt{2 \pi}} I_{\left\{t>\frac{1}{2 a}\right\}}+\infty I_{\left\{t \leq \frac{1}{2 a}\right\}} \text { as } u_{2} \rightarrow 0 .
\end{aligned}
$$

We have (21) from (8) and (52)-(54).

Proof of Theorem 3 . We have that

$$
\operatorname{LPE}_{X_{t}}(u)=\mathrm{E}\left(X_{t} I_{\left\{X_{t} \leq u\right\}}\right)=\mathrm{E}\left(\mathrm{E}\left(X_{t} I_{\left\{X_{t} \leq u\right\}} \mid Y_{t}\right)\right)
$$

with $Y_{t}$ defined in (29). Let

$$
\bar{g}\left(Y_{t}\right)=\mathrm{E}\left(X_{t} I_{\left\{X_{t} \leq u\right\}} \mid Y_{t}\right)
$$

Then

$$
\begin{aligned}
& \bar{g}\left(Y_{t}\right)=\mathrm{E}\left(\left(\int_{0}^{t} \mu(s) d s\right) I_{\left\{X_{t} \leq u\right\}} \mid Y_{t}\right)+Y_{t} g\left(Y_{t}\right)= \\
= & \mu t e^{-\lambda t} I_{\left\{Y_{t} \leq u-\mu t\right\}}+\mathrm{E}\left((\mu \tau+\widehat{\mu}(t-\tau)) I_{\left\{X_{t} \leq u, \tau \leq t\right\}} \mid Y_{t}\right)+Y_{t} g\left(Y_{t}\right)= \\
= & \mu t e^{-\lambda t} I_{\left\{Y_{t} \leq u-\mu t\right\}}+Y_{t} g\left(Y_{t}\right)+\mathrm{p} \mu t\left(1-e^{-\lambda t}\right) I_{\left\{Y_{t} \leq u-\mu t\right\}}+ \\
+ & t \sum_{j=1}^{m} \mathrm{p}_{j} \mu_{j}\left[\mathrm{P}\left(\tau \leq \min \left\{t, \frac{u-\mu_{j} t-Y_{t}}{\mu-\mu_{j}}\right\} \mid Y_{t}\right) I_{\left\{Y_{t} \leq u-\mu_{j} t \mu_{j}<\mu\right\}}+\right. \\
+ & \left.\left(1-e^{-\lambda t}\right) I_{\left\{Y_{t} \leq u-\mu t, \mu_{j}=\mu\right\}}\right]+\sum_{j=1}^{m} \mathrm{p}_{j}\left(\mu-\mu_{j}\right) \times \\
& \times \mathrm{E}\left(\tau I_{\left\{\tau \leq \min \left\{t, \frac{u-\mu_{j} t-Y_{t}}{\mu-\mu_{j}}\right\}\right.} \mid Y_{t}\right) I_{\left\{Y_{t} \leq u-\mu_{j} t, \mu_{j}<\mu\right\}^{\prime}}
\end{aligned}
$$


where $g\left(Y_{t}\right)$ is assigned by (30). Using (32), we get that

$$
\begin{aligned}
& \bar{g}\left(Y_{t}\right)=\mu t\left(\mathrm{p}+e^{-\lambda t} \sum_{j=1}^{m} \mathrm{p}_{j}\right) I_{\left\{Y_{t} \leq u-\mu t\right\}}+Y_{t} g\left(Y_{t}\right)+ \\
& +t \sum_{j=1}^{m} \mathrm{p}_{j} \mu_{j}\left[\left(1-e^{-\lambda t}\right) I_{\left\{Y_{t} \leq u-\mu t\right\}}+\left(1-e^{\lambda \frac{Y_{t}+\mu_{j} t-u}{\mu-\mu_{j}}}\right) \times\right. \\
& \left.\times I_{\left\{u-\mu t<Y_{t} \leq u-\mu_{j} t, \mu_{j}<\mu\right\}}\right]+\sum_{j=1}^{m} \mathrm{p}_{j}\left(\mu-\mu_{j}\right) \times \\
& \times \mathrm{E}\left(\tau I_{\left.\left\{\tau \leq \min \left\{t, \frac{u-\mu_{j} t-Y_{t}}{\mu-\mu_{j}}\right\}\right\}^{\mid Y_{t}}\right) I_{\left\{Y_{t} \leq u-\mu_{j} t, \mu_{j}<\mu\right\}} .}\right.
\end{aligned}
$$

Next, the conditional expectation

$$
\begin{aligned}
& \mathrm{E}\left(\tau I_{\left\{\tau \leq \min \left\{t, \frac{u-\mu_{j} t-Y_{t}}{\mu-\mu_{j}}\right\}\right\}^{\mid}} \mid Y_{t}\right) I_{\left\{Y_{t} \leq u-\mu_{j} t\right\}}= \\
= & \mathrm{E}\left(\tau I_{\{\tau \leq t\}} \mid Y_{t}\right) I_{\left\{Y_{t} \leq u-\mu t\right\}}+\mathrm{E}\left(\tau I_{\left\{\tau \leq \frac{u-\mu_{j} t-Y_{t}}{\mu-\mu_{j}}\right\}} \mid Y_{t}\right) I_{\left\{u-\mu t<Y_{t} \leq u-\mu_{j} t\right\}}= \\
= & \left(\frac{1}{\lambda}\left(1-e^{-\lambda t}\right)-t e^{-\lambda t}\right) I_{\left\{Y_{t} \leq u-\mu t\right\}}+\left(\frac{1}{\lambda}+\left(\frac{\mu_{j} t-u}{\mu-\mu_{j}}-\frac{1}{\lambda}\right) \times\right. \\
\times & \left.e^{\frac{\lambda\left(Y_{t}+\mu_{j} t-u\right)}{\mu-\mu_{j}}}+\frac{Y_{t}}{\mu-\mu_{j}} e^{\frac{\lambda\left(Y_{t}+\mu_{j} t-u\right)}{\mu-\mu_{j}}}\right) I_{\left\{u-\mu t<Y_{t} \leq u-\mu_{j} t\right\}}
\end{aligned}
$$

for $\mu_{j}<\mu$ since

$$
\lambda \int_{0}^{\chi} x e^{-\lambda x} d x=-\chi e^{-\lambda \chi}+\frac{1}{\lambda}\left(1-e^{-\lambda \chi}\right) .
$$

Substituting (57) in (56), we get that

$$
\begin{aligned}
& \bar{g}\left(Y_{t}\right)=Y_{t} g\left(Y_{t}\right)+C_{1} I_{\left\{Y_{t} \leq u-\mu t\right\}}+\sum_{j=1}^{m} \mathrm{p}_{j}\left\{t \mu_{j}\left(1-e^{\lambda \frac{Y_{t}+\mu_{j} t-u}{\mu-\mu_{j}}}\right) \times\right. \\
& \times I_{\left\{u-\mu t<Y_{t} \leq u-\mu_{j} t\right\}}+\left(\mu-\mu_{j}\right)\left[C_{2} I_{\left\{Y_{t} \leq u-\mu t\right\}}+\right. \\
& \left.\left.+\left(\frac{1}{\lambda}+\left(C_{3 j}+\frac{Y_{t}}{\mu-\mu_{j}}\right) e^{\frac{\lambda\left(Y_{t}+\mu_{j} t-u\right)}{\mu-\mu_{j}}}\right) I_{\left\{u-\mu t<Y_{t} \leq u-\mu_{j} t\right\}}\right]\right\} I_{\left\{\mu_{j}<\mu\right\}}
\end{aligned}
$$

with

$$
\begin{aligned}
& C_{1}=t\left(\mu\left[\mathrm{p}+e^{-\lambda t} \sum_{j=1}^{m} \mathrm{p}_{j}\right]+\left(1-e^{-\lambda t}\right) \sum_{j=1}^{m} \mathrm{p}_{j} \mu_{j}\right), \\
& C_{2}=\frac{1}{\lambda}\left(1-e^{-\lambda t}\right)-t e^{-\lambda t}, \\
& C_{3 j}=\frac{\mu_{j} t-u}{\mu-\mu_{j}}-\frac{1}{\lambda} .
\end{aligned}
$$


It follows from (58) that

$$
\begin{aligned}
& \bar{g}\left(Y_{t}\right)=Y_{t} g\left(Y_{t}\right)+C_{1} I_{\left\{Y_{t} \leq u-\mu t\right\}}+\sum_{j=1}^{m} \mathrm{p}_{j}\left\{\left[\left(\mu-\mu_{j}\right)\left(C_{2}-\frac{1}{\lambda}\right)-t \mu_{j}\right] \times\right. \\
& \times I_{\left\{Y_{t} \leq u-\mu t\right\}}+e^{\frac{\lambda\left(\mu_{j} t-u\right)}{\mu-\mu_{j}}}\left(t \mu_{j}-C_{3 j}\left(\mu-\mu_{j}\right)\right) e^{\frac{\lambda Y_{t}}{\mu-\mu_{j}}} I_{\left\{Y_{t} \leq u-\mu t\right\}}-e^{\frac{\lambda\left(\mu_{j} t-u\right)}{\mu-\mu_{j}}} \times \\
& \times Y_{t} e^{\frac{\lambda Y_{t}}{\mu-\mu_{j}}} I_{\left\{Y_{t} \leq u-\mu t\right\}}+\left(t \mu_{j}+\frac{\left(\mu-\mu_{j}\right)}{\lambda}\right) I_{\left\{Y_{t} \leq u-\mu_{j} t\right\}}+ \\
& +e^{\frac{\lambda\left(\mu_{j} t-u\right)}{\mu-\mu_{j}}}\left(\left(\mu-\mu_{j}\right) C_{3 j}-t \mu_{j}\right) e^{\frac{\lambda Y_{t}}{\mu-\mu_{j}}} I_{\left\{Y_{t} \leq u-\mu_{j} t\right\}}+ \\
& \left.+e^{\frac{\lambda\left(\mu_{j} t-u\right)}{\mu-\mu_{j}}} Y_{t} e^{\frac{\lambda Y_{t}}{\mu-\mu_{j}}} I_{\left\{Y_{t} \leq u-\mu_{j} t\right\}}\right\} I_{\left\{\mu_{j}<\mu\right\}}
\end{aligned}
$$

and substituting $g\left(Y_{t}\right)$ in (59) from (34), we establish that

$$
\begin{aligned}
& \bar{g}\left(Y_{t}\right)=Y_{t} I_{\left\{Y_{t} \leq u-\mu t\right\}}+C_{1} I_{\left\{Y_{t} \leq u-\mu t\right\}}+\sum_{j=1}^{m} \mathrm{p}_{j}\left\{\left[\left(\mu-\mu_{j}\right)\left(C_{2}-\frac{1}{\lambda}\right)-t \mu_{j}\right] \times\right. \\
& \times I_{\left\{Y_{t} \leq u-\mu t\right\}}+e^{\frac{\lambda\left(\mu_{j} t-u\right)}{\mu-\mu_{j}}}\left(t \mu_{j}-C_{3 j}\left(\mu-\mu_{j}\right)\right) e^{\frac{\lambda Y_{t}}{\mu-\mu_{j}}} I_{\left\{Y_{t} \leq u-\mu t\right\}}+ \\
& +\left(t \mu_{j}+\frac{\left(\mu-\mu_{j}\right)}{\lambda}\right) I_{\left\{Y_{t} \leq u-\mu_{j} t\right\}}+Y_{t} I_{\left\{Y_{t} \leq u-\mu_{j} t\right\}}+ \\
& \left.+e^{\frac{\lambda\left(\mu_{j} t-u\right)}{\mu-\mu_{j}}}\left(\left(\mu-\mu_{j}\right) C_{3 j}-t \mu_{j}\right) e^{\frac{\lambda Y_{t}}{\mu-\mu_{j}}} I_{\left\{Y_{t} \leq u-\mu_{j} t\right\}}\right\} I_{\left\{\mu_{j}<\mu\right\}} .
\end{aligned}
$$

Let

$$
\begin{aligned}
& C_{4 j}=\left(\mu-\mu_{j}\right)\left(C_{2}-\frac{1}{\lambda}\right)-t \mu_{j}, \\
& C_{5 j}=e^{\frac{\lambda\left(\mu_{j} t-u\right)}{\mu-\mu_{j}}}\left(t \mu_{j}-C_{3 j}\left(\mu-\mu_{j}\right)\right), \\
& C_{6 j}=t \mu_{j}+\frac{\left(\mu-\mu_{j}\right)}{\lambda}, \\
& C_{7 j}=e^{\frac{\lambda\left(\mu_{j} t-u\right)}{\mu-\mu_{j}}}\left(\left(\mu-\mu_{j}\right) C_{3 j}-t \mu_{j}\right) .
\end{aligned}
$$

Then since $\operatorname{LPE}_{X_{t}}(u)=\mathrm{E} \bar{g}\left(Y_{t}\right)$, we have that

$$
\begin{aligned}
& \operatorname{LPE}_{X_{t}}(u)=\mathrm{E}\left(Y_{t} I_{\left\{Y_{t} \leq u-\mu t\right\}}\right)+C_{1} \mathrm{P}\left(Y_{t} \leq u-\mu t\right)+ \\
& +\sum_{j=1}^{m} \mathrm{p}_{j} I_{\left\{\mu_{j}<\mu\right\}}\left\{C_{4 j} \mathrm{P}\left(Y_{t} \leq u-\mu t\right)+C_{5 j} \mathrm{E}\left(e^{\frac{\lambda Y_{t}}{\mu-\mu_{j}}} I_{\left\{Y_{t} \leq u-\mu t\right\}}\right)+\right. \\
& \left.+C_{6 j} \mathrm{P}\left(Y_{t} \leq u-\mu_{j} t\right)+\mathrm{E}\left(Y_{t} I_{\left\{Y_{t} \leq u-\mu_{j} t\right\}}\right)+C_{7 j} \mathrm{E}\left(e^{\frac{\lambda Y_{t}}{\mu-\mu_{j}}} I_{\left\{Y_{t} \leq u-\mu_{j} t\right\}}\right)\right\} .
\end{aligned}
$$


Using (38), we get that

$$
\begin{aligned}
& \frac{\operatorname{LPE}_{X_{t}}(u) \Gamma(a t)}{a^{a t}}=\mathfrak{I}\left(a t, 0,-\frac{\theta}{\sigma^{\prime}}, \frac{u-\mu t}{\sigma}\right)+C_{1} \mathfrak{I}\left(a t-1,0,-\frac{\theta}{\sigma^{\prime}}, \frac{u-\mu t}{\sigma}\right)+ \\
& +\sum_{j=1}^{m} \mathrm{p}_{j} I_{\left\{\mu_{j}<\mu\right\}}\left\{C_{4 j} \mathfrak{I}\left(a t-1,0,-\frac{\theta}{\sigma}, \frac{u-\mu t}{\sigma}\right)+\right. \\
& +C_{5 j} \mathfrak{I}\left(a t-1, w_{j}, z_{j}, \frac{u-\mu t}{\sigma}\right)+C_{6 j} \mathfrak{I}\left(a t-1,0,-\frac{\theta}{\sigma}, \frac{u-\mu_{j} t}{\sigma}\right)+ \\
& \left.+\mathfrak{I}\left(a t, 0,-\frac{\theta}{\sigma}, \frac{u-\mu_{j} t}{\sigma}\right)+C_{7 j} \mathfrak{I}\left(a t-1, w_{j}, z_{j}, \frac{u-\mu_{j} t}{\sigma}\right)\right\} .
\end{aligned}
$$

Combining together (22), (23)-(27) and (60), we get (28).

Funding: This research received no external funding.

Institutional Review Board Statement: Not applicable.

Informed Consent Statement: Not applicable.

Data Availability Statement: Not applicable.

Acknowledgments: I would like to thank the anonymous referees whose suggestions have improved the economic impact of the work.

Conflicts of Interest: The authors declare no conflict of interest.

\section{References}

Aguilar, Jean-Philippe. 2020. Some pricing tools for the variance gamma model. International Journal of Theoretical and Applied Finance 23: 2050025. [CrossRef]

Almendral, Ariel, and Cornelis W. Oosterlee. 2007. On American Options Under the Variance Gamma Process. Applied Mathematical Finance 14: 131-52. [CrossRef]

Ano, Katsunori, and Roman V. Ivanov. 2016. On exact pricing of FX options in multivariate time-changed Lévy models. Review of Derivatives Research 19: 201-16.

Armenti, Yannick, Stéphane Crépey, Samuel Drapeau, and Antonis Papapantoleon. 2018. Multivariate shortfall risk allocation and systemic risk. SIAM Journal on Financial Mathematics 9: 90-126. [CrossRef]

Avramidis, Athanassios N., Pierre L. Ecuyer, and Pierre-Alexandre Tremblay. 2003. Efficient simulation of gamma and variance-gamma processes. Paper presented at 2003 Winter Simulation Conference, New Orleans, LA, USA, December 7-10. Edited by Chick Snchez Ferrin, S. Chick, P. J. Sánchez, D. Ferrin, D. J. Morrice and Ying-chao Hung, pp. 319-26.

Barone Adesi, Giovanni. 2016. VaR and CVaR Implied in Option Prices. Journal of Risk and Financial Management 9: 2. [CrossRef]

Bateman, Harry, and Arthur Erdélyi. 1953. Higher Transcendental Functions. New York: McGraw-Hill.

Beibel, Martin, and Hans Rudolf Lerche. 1997. A new look at optimal stopping problems related to mathematical finance. Statistica Sinica 7: 93-108.

Bernanke, Ben S., and Alan S. Blinder. 1992. The Federal Funds Rate and the Channels of Monetary Transmission. American Economic Review 82: 901-21.

Brent, Richard P. 1973. Algorithms for Minimization without Derivatives. Hoboken: Prentice-Hall.

Cai, Zongwu, and Xian Wang. 2008. Nonparametric estimation of conditional var and expected shortfall. Journal of Econometrics 147: 120-30. [CrossRef]

Carr, Peter, and Dilip Madan. 1999. Option valuation using the fast Fourier transform. Journal of Computational Finance 2: 61-73. [CrossRef]

Chen, Song Xi, and Cheng Yong Tang. 2005. Nonparametric inference of value-at-risk for dependent financial returns. Journal of Financial Econometrics 3: 227-55. [CrossRef]

Christoffersen, Peter, and Denis Pelletier. 2004. Backtesting Value-at-Risk: A Duration-Based Approach. Journal of Financial Econometrics 2: 84-108. [CrossRef]

Chun, So Yeon, Alexander Shapiro, and Stan Uryasev. 2012. Conditional Value-at-Risk and Average Value-at-Risk: Estimation and Asymptotics. Operations Research 60: 739-56. [CrossRef]

Cook, Timothy, and Thomas Hahn. 1989. The effect of changes in the federal funds rate target on market interest rates in the 1970s. Journal of Monetary Economics 24: 331-51. [CrossRef]

Daal, Elton A., and Dilip B. Madan. 2005. An Empirical Examination of the Variance-Gamma Model for Foreign Currency Options. The Journal of Business 78: 2121-52. [CrossRef] 
Drapeau, Samuel, Michael Kupper, and Antonis Papapantoleon. 2014. A Fourier approach to the computation of CVaR and optimized certainty equivalents. Journal of Risk 16: 3-29. [CrossRef]

Febrer, Pedro, and João Guerra. 2021. Residue Sum Formula for Pricing Options under the Variance Gamma Model. Mathematics 9: 1143. [CrossRef]

Finlay, Richard, and Eugene Seneta. 2006. Stationary-increment student and variance-gamma processes. Journal of Applied Probability 43: 441-53. [CrossRef]

Flora, Maria, and Tiziano Vargiolu. 2020. Price dynamics in the European Union Emissions Trading System and evaluation of its ability to boost emission-related investment decisions. European Journal of Operational Research 280: 383-94. [CrossRef]

Fu, Michael C. 2007. Variance-Gamma and Monte-Carlo. In Advances in Mathematical Finance. Edited by Michael C. Fu, Robert A. Jarrow, Ju-Yi Yen and Robert J. Elliott. Boston: Birkhauser, pp. 21-34.

Fuh, Cheng-Der, Kwok Wah Remus Ho, Inchi Hu, and Ren-Her Wang. 2012. Option Pricing with Markov Switching. Journal of Data Science 10: 483-509. [CrossRef]

Göncü, Ahmet, Mehmet Oğuz Karahan, and Tolga Umut Kuzubaş. 2016. A comparative goodness-of-fit analysis of distributions of some Lévy processes and Heston model to stock index returns. The North American Journal of Economics and Finance 36: 69-83. [CrossRef]

Gradshteyn, Izrail Solomonovich, and Iosif Moiseevich Ryzhik. 2007. Table of Integrals, Series and Products. New York: Academic Press.

Hoyyi, A., D. A. I. Maruddani, and R. Rahmawati. 2021. Variance gamma for stock model performance with excess kurtosis. Journal of Physics: Conference Series 1943: 012146. [CrossRef]

Ivanov, Roman V. 2018. A credit-risk valuation under the variance-gamma asset return. Risks 6: 58. [CrossRef]

Ivanov, Roman V. 2021. The downside and upside beta valuation in the variance-gamma model. International Journal of Analysis and Applications 19: 319-40. [CrossRef]

Ivanov, Roman V., and Grigory Temnov. 2016. On the conditional moment-generating function of a three-factor variance gamma based process and its application to forward and futures pricing. Markov Processes and Related Fields 22: 737-58.

Keçeci, Neslihan Fidan, Viktor Kuzmenko, and Stan Uryasev. 2016. Portfolios Dominating Indices: Optimization with Second-Order Stochastic Dominance Constraints vs. Minimum and Mean Variance Portfolios. Journal of Risk and Financial Management 9: 11. [CrossRef]

Linders, Daniël, and Ben Stassen. 2016. The multivariate variance gamma model: Basket option pricing and calibration. Quantitative Finance 16: 555-72. [CrossRef]

Luciano, Elisa, and Wim Schoutens. 2016. A multivariate jump-driven financial asset model. Quantitative Finance 6: 385-402. [CrossRef]

Luciano, Elisa, Marina Marena, and Patrizia Semeraro. 2016. Dependence calibration and portfolio fit with factor-based subordinators. Quantitative Finance 16: 1037-52. [CrossRef]

Madan, Dilip B., and Eugene Seneta. 1990. The Variance Gamma (V.G.) Model for Share Market Returns. Journal of Business 63: 511-24. [CrossRef]

Madan, Dilip B., and Frank Milne. 1991. Option pricing with VG martingale components. Mathematical Finance 1: 39-55. [CrossRef]

Madan, Dilip B., Peter P. Carr, and Eric C. Chang. 1998. The Variance Gamma Process and Option Pricing. European Finance Review 2: 79-105. [CrossRef]

Mafusalov, Alexander, and Stan Uryasev. 2016. CVaR (Superquantile) Norm: Stochastic Case. European Journal of Operational Research 249: 200-8. [CrossRef]

Ming Chen, James. 2018. On Exactitude in Financial Regulation: Value-at-Risk, Expected Shortfall, and Expectiles. Risks 6: 61. [CrossRef]

Moosbrucker, Thomas. 2006. Explaining the correlation smile using variance gamma distributions. Journal of Fixed Income 16: 71-87. [CrossRef]

Mozumder, Sharif, Ghulam Sorwar, and Kevin Dowd. 2015. Revisiting variance gamma pricing: An application to s\&p500 index options. International Journal of Financial Engineering 2: 1-24.

Nakamura, Emi, and Jón Steinsson. 2018. High-Frequency Identification of Monetary Non-Neutrality: The Information Effect. The Quarterly Journal of Economics 133: 1283-330. [CrossRef]

Nitithumbundit, Thanakorn, and Jennifer S. K. Chan. 2020. ECM Algorithm for Auto-Regressive Multivariate Skewed Variance Gamma Model with Unbounded Density. Methodology and Computing in Applied Probability 22: 1169-91. [CrossRef]

Novikov, Alexander A., and Albert Shiryaev. 2009. On a stochastic version of the trading rule "Buy and Hold". Statistics E Decisions 26: 289-302.

Press, William H., Brian P. Flannery, Saul A. Teukolsky, and William T. Vetterling. 2007. Numerical Recipes. Cambridge: Cambridge University Press.

Ramsey, A. Ford, and Barry K. Goodwin. 2019. Value-at-Risk and Models of Dependence in the U.S. Federal Crop Insurance Program. Journal of Risk and Financial Management 12: 65. [CrossRef]

Rathgeber, Andreas W., Johannes Stadler, and Stefan Stöckl. 2016. Modeling share returns-An empirical study on the Variance Gamma model. Journal of Economics and Finance 40: 653-82. [CrossRef]

Rémillard, Bruno, Alexandre Hocquard, Hugo Lamarre, and Nicolas Papageorgiou. 2017. Option Pricing and Hedging for Discrete Time Regime-Switching Models. Modern Economy 8: 1005-32. [CrossRef]

Rockafellar, R. Tyrrell, and Stanislav Uryasev. 2000. Optimization of conditional value-at-risk. Journal of Risk 2: 21-41. [CrossRef] 
Scaillet, Olivier. 2005. Nonparametric estimation of conditional expected shortfall. Insurance and Risk Management Journal 74: 639-60. Shiryaev, Albert N. 1963. On optimum methods in quickest detection problems. Theory of Probability and its Applications 8: 22-46. [CrossRef]

Srivastava, Hari M., and Per Wennerberg Karlsson. 1985. Multiple Gaussian Hypergeometric Series. New York: Wiley.

Stoer, Josef, and Roland Bulirsch. 2002. Introduction to Numerical Analysis. Berlin: Springer.

Wallmeier, Martin, and Martin Diethelm. 2012. Multivariate downside risk: Normal versus variance gamma. Journal of Futures Markets 32: 431-58. [CrossRef]

Yao, David D., Qing Zhang, and Xun Yu Zhou. 2006. A Regime-Switching Model for European Options. In Stochastic Processes, Optimization, and Control Theory: Applications in Financial Engineering, Queueing Networks, and Manufacturing Systems. Edited by Houmin Yan, G. George Yin and Qing Zhang. Boston: Springer, pp. 281-300.

Yin, G. George, and Chao Zhu. 2010. Hybrid Switching Diffusions. New York: Springer.

Zhang, Qing, and Xun Yu Zhou. 2009. Valuation of Stock Loans with Regime Switching. SIAM Journal on Control and Optimization 48: 1229-50. [CrossRef] 